UNIVERSIDADE DE SÃO PAULO

FACULDADE DE MEDICINA DE RIBEIRÃO PRETO

ANDERSON LUIS DO NASCIMENTO

Estudo morfométrico do pedículo cervical

Ribeirão Preto 
ANDERSON LUIS DO NASCIMENTO

\section{Estudo morfométrico do pedículo cervical}

Dissertação apresentada à Faculdade de Medicina de Ribeirão Preto da Universidade de São Paulo, para obtenção do título de Mestre em Ortopedia.

Área de concentração: Ortopedia, Traumatologia e Reabilitação do Aparelho Locomotor.

Orientador: Prof. Dr. Carlos Fernando Pereira da Silva Herrero

Ribeirão Preto 
Autorizo a reprodução e divulgação total ou parcial deste trabalho, por qualquer meio convencional ou eletrônico, para fins de estudo e pesquisa, desde que citada a fonte.

Nascimento, Anderson Luis do

Estudo morfométrico do pedículo cervical $\backslash$ Anderson Luis do Nascimento - Ribeirão Preto, 2016.

57 p.: il.; $30 \mathrm{~cm}$.

Dissertação de Mestrado, apresentada à Faculdade de Medicina de Ribeirão Preto/USP. Área de concentração: Neurologia.

Orientador: Carlos Fernando Pereira da Silva Herrero.

1.Tomografia Computadorizada. 2. Pedículo. 3.Anatomia. 4.Coluna cervical.

CDD 616.12 


\section{FOLHA DE APROVAÇÃO}

Anderson Luis do Nascimento

Estudo morfométrico do pedículo cervical.

Dissertação apresentada à Faculdade de Medicina de Ribeirão Preto da Universidade de São Paulo, para obtenção do título de Mestre em Ortopedia.

Área de concentração: Ortopedia, Traumatologia e Reabilitação do Aparelho Locomotor

Aprovado em:

Banca Examinadora

Prof. Dr.

Instituição:

Assinatura:

Prof. Dr.

Instituição:

Assinatura:

Prof. Dr.

Instituição:

Assinatura: 


\section{DEDICATÓRIA}

A Deus, pelo DOM da vida e por iluminar e guiar minha jornada.

À minha mãe Ana, por ser o meu maior exemplo de caridade, paciência e amor ao próximo. Ao meu paiJerson, por me ensinar a ser determinado e trabalhador. Devo tudo que sou a eles.

À minha esposa Bárbara, que me incentivou "indiretamente" a ser persistente e muitas vezes teimoso, sempre me dando o apoio necessário para realizar meu trabalho. Às minhas filhas Manuela e Sofia, que são os principais motivos para me tornar uma pessoa mais evoluída e tentar ser um exemplo. 


\section{AGRADECIMENTOS}

Ao Prof. Dr. Fernando Herrero (Carlos Fernando Pereira da Silva Herrero) meu amigo e orientador, pelo exemplo de pessoa e profissional, pela ajuda incondicional, pelos conselhos e ensinamentos, e sobretudo pelo carinho e amizade.

Ao Prof. Dr. Helton Luiz Aparecido Defino, pelo incentivo no passo inicial desse trabalho, pela oportunidade de ser seu aluno e de integrar na residência médica a equipe de coluna do Hospital das Clínicas de Ribeirão Preto e pelo continuo incentivo à pesquisa.

Ao Dr. Mario Bressan Neto meu parceiro de residência em ortopedia e também em cirurgia de coluna, pela amizade e companheirismo, pela ajuda durante o desenvolvimento do meu trabalho e principalmente pelas risadas mesmo nos dias mais difíceis da residência.

A equipe da cirurgia de coluna do Hospital das Clínicas de Ribeirão Preto : Dr. Herton Costa pelas dicas no mestrado; Dr Rafael Sugino pela parceria; Dr. Narcélio Mendes pela ajuda nas medidas tomográficas do trabalho.

Aos professores e funcionários da pós graduação do programa de ciências da saúde aplicadas ao aparelho locomotor da FMRP-USP, em especial ao Prof. Dr Marcelo Riberto e à secretária Rita de Cássia, pela paciência, pelas orientações e pela atenção que sempre me ofereceram.

Aos amigos e funcionários do Hospital das Clínicas de Ribeirão Preto e do Departamento de Biomecânica, Medicina e Reabilitação do Aparelho Locomotor (secretários, enfermeiros, auxiliares de enfermagem, instrumentadores e circulantes de sala cirúrgica ) que durante esse trabalho, me deram palavras de incentivo e me apoiaram. 
"A dévida é a princípia da sabedaria." Aristateles 


\section{RESUMO}

NASCIMENTO, A. L.Estudo morfométrico do pedículo cervical. 2016.57f. Dissertação (Mestrado) - Faculdade de Medicina de Ribeirão Preto, Universidade de São Paulo, Ribeirão Preto, SP, 2016.

A inserção do parafuso pedicular cervical é um procedimento cirúrgico tecnicamente desafiador. Assim, o amplo conhecimento da morfologia das vértebras cervicais, particularmente do pedículo, é essencial para minimizar o risco de ferimentos graves a estruturas neurovasculares. $\mathrm{O}$ objetivo deste estudo foi conduzir uma avaliação detalhada de exames de tomografia computadorizada (TC) na população brasileira do ponto de entrada, trajetória, e dimensões dos pedículos da coluna cervical. Duzentos pacientes consecutivos foram retrospectivamente avaliados utilizando a TC da coluna cervical, com a reconstrução da imagem de cada vértebra no plano axial com $2 \mathrm{~mm}$, e no plano sagital com $3 \mathrm{~mm}$. Os parâmetros no plano axial incluíram a espessura pedicular (EP), o comprimento pedicular axial (CPA), o ângulo pedicular transverso (APT) e a distância do ponto de entrada ao ângulo entre a lâmina e o processo espinhoso (DPE). As medidas no plano sagital envolveram a altura pedicular (AP) e o ângulo pedicular sagital (APS). A EP e AP média são menores nos pacientes do sexo feminino do que nos pacientes do sexo masculino em todas as vertebras cervicais, porém não houve diferença significativa do APT entre os sexos. O APS variou de $15,2^{\circ}$ a $23,7^{\circ}$. Os valores médios do CPA e DPE apresentaram uma tendência a diminuir de proximal para distal na coluna cervical. A EP encontrada foi menor que $4 \mathrm{~mm}$ em $7,5 \%$ dos pacientes do sexo masculino (C3) e em $25 \%$ dos pacientes do sexo feminino (C3), e menores que $4,5 \mathrm{~mm}$ em $20 \%$ dos pacientes do sexo masculino (C3) e em $66 \%$ dos pacientes do sexo feminino (C3). A confiabilidade intra e interobservador foram muito boas para a medida tomográfica da EP, e boa para a AP. Para o CPA, a confiabilidade intraobservador foi boa, porém a confiabilidade interobservador variou de moderada a boa. Considerando o APT e APS, a confiabilidade intraobservador foi boa, mas a confiabilidade interobservador moderada para o APT e fraca para o APS. As medidas do DPE evidenciaram fraca confiabilidade intraobservador e fraca ou moderada confiabilidade interobservador. Nossos resultados apresentaram tendência similar aos de estudos prévios, no entanto a frequência de pacientes com a EP $<4,5 \mathrm{~mm}$ em nossa população é maior, sugerindo um risco aumentado durante a tentativa de realizar a técnica transpedicular.

Descritores: anatomia, coluna cervical, tomografia computadorizada, pedículo 


\begin{abstract}
NASCIMENTO, A. L. Morphometric study of the cervical pedicle. 2016.57f. Dissertation (Master) - School of Medicine of RibeirãoPreto, University of São Paulo, RibeirãoPreto, SP, 2016.
\end{abstract}

Pedicular screw insertion is a technically challenged surgical procedure. Thus, extensive knowledge of the morphology of the cervical vertebra, primarily the pedicle, is crucial to reduce severe damage to neurovascular structures. The goal of this study was to conduct a detailed computed tomography (CT) assessment in the Brazilian population of the screw starting point, trajectory, and dimensions of pedicle in the cervical spine. Two hundred consecutive patients were retrospectively evaluated using cervical spine CT, with imaging reconstruction of each cervical vertebra in the axial plane with $2 \mathrm{~mm}$, and in sagittal reconstructions with $3 \mathrm{~mm}$. Parameters in axial plane included the pedicle width (PW), pedicle axis length (PAL), pedicle transverse angle (PTA), and the distance from the entry point to the point between the lamina and spinous process (DEP). Measurements in the sagittal plane involved the pedicle height (PH) and the pedicle sagittal angle (PSA). The mean PW and PH were smaller in females than in males in all cervical vertebrae, but there were no significant differences of PTA among genders. PSA ranged from $15.2^{\circ}$ to $23.7^{\circ}$. Mean values of PAL and DEP had a tendency to decrease from the proximal to distal cervical vertebrae. PW was $<4 \mathrm{~mm}$ in $7.5 \%$ of men (C3) and $25 \%$ of women (C3), and $<4.5 \mathrm{~mm}$ in $20 \%$ (C3 male) and $66 \%$ (C3 female). The intra- and inter-observer reliability were very good for the tomographic measurement of PW, and good for PH. For PAL, the intraobserver reliability was good, but the interobserver reliability varied from moderate to good. Considering PTA and PSA, the intraobserver reliability was good, but the interobserver reliability moderate for PTA and poor or fair for PSA. DEP measurements showed poor intraobserver reliability, and poor or moderate interobserver reliability. Our results presented similar trend of previous studies, but the frequency of patients with $\mathrm{PW}<4.5 \mathrm{~mm}$ in our population is higher, suggesting an increased risk during the attempting of transpedicular screw technique.

Keywords: anatomy, cervical spine, computed tomography, pedicle 


\section{LISTA DE ILUSTRAÇÕES}

FIGURA 1 - Cortes transversais do embrião. A e B, note os somitos recém formados. Em C, observe a diferenciação do somito em dermomiótomo e esclerótomo, e em $\mathrm{D}$, a diferenciação do demomiótomo em demátomo e miótomo............................................ 15

FIGURA 2 - Vértebras Cervicais [C4 e C7] ............................................................. 16

FIGURA 3 - Vértebras Cervicais (C2-T1) agrupadas - vista lateral direita............ 18

FIGURA 4 - Imagem da reconstrução axial de tomografia computadorizada ilustrando as medidas realizadas neste plano.................................... 28

FIGURA 5 - Imagem da reconstrução sagital de tomografia computadorizada ilustrando as medidas realizadas neste plano.................................. 28 


\section{LISTA DE TABELAS}

Tabela 1 - $\quad$ Parâmetros medidos nas imagens axiais da TC ...................................... 27

Tabela 2 - $\quad$ Parâmetros medidos nas imagens sagitais da TC................................. 27

Tabela 3 - $\quad$ Resumo dos parâmetros lineares e angulares dos pedículos cervicais.... 30

Tabela 4 - $\quad$ Distribuição da largura do pedículo na amostra masculina.................... 33

Tabela 5 - $\quad$ Distribuição da largura do pedículo na amostra feminina...................... 34

Tabela 6 - Os coeficientes de correlação intraclasse (CCI) com o respectivo intervalo de confiança de 95\% (IC 95\%) para estimativa de confiabilidade intra e interobservador.................................................. 


\section{LISTA DE GRÁFICOS}

Gráfico 1 - Ilustrando a variação da espessura pedicular média em milímetros nos níveis vertebrais cervicais estudados em ambos os sexos.

Gráfico 2 - Ilustrando a variação do comprimento pedicular axial médio em milímetros nos níveis vertebrais cervicais estudados em ambos os sexos.... 35

Gráfico 3 - Ilustrando a variação da distância em milímetros do ponto de entrada ao ângulo entre a lâmina e o processo espinhoso média nos níveis vertebrais cervicais estudados em ambos os sexos.

Gráfico 4 - Ilustrando a variação angular média em graus do pedículo na posição transversal nos níveis vertebrais cervicais estudados em ambos os sexos.... 37

Gráfico 5 - Ilustrando a variação da altura pedicular média em milímetros nos níveis vertebrais cervicais estudados em ambos os sexos.

Gráfico 6 - Ilustrando a variação angular média em graus do pedículo na posição sagital, nos níveis vertebrais cervicais estudados em ambos os sexos. 


\section{LISTA DE ABREVIATURAS}

AP- - altura pedicular

APS - ângulo pedicular sagital

APT - ângulo pedicular transverso

CCI - coeficiente de correlação interclasses

CPA - comprimento pedicular axial

C1 - primeira vertebral cervial

C2 - segunda vertebral cervial

C3 - terceira vertebral cervial

C4 - quarta vertebral cervial

C5 - quinta vertebral cervial

C6 - sexta vertebral cervial

C7 - sétima vertebral cervial

DISH -doença hiperostótica familiar

DPE - distância do ponto de entrada

EP - espessura pedicular

F -feminino

FMRP - faculdade de medicina de Ribeirão Preto

IC -intervalo de confiança

M - masculino

mm - milímetros

TC - tomografia computadorizada

T1 - primeira vertebra torácica

USP - Universidade de São Paulo 
1 INTRODUÇÃ $O$............................................................................................................. 13

1.1 Embriologia coluna cervical ................................................................................ 14

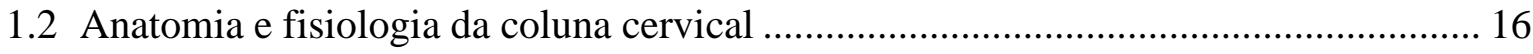

1.3 Técnicas cirúrgicas coluna cervical...................................................................... 18

1.4 Método tomográfico de imagem na coluna cervical ................................................ 20

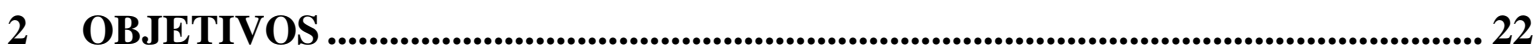

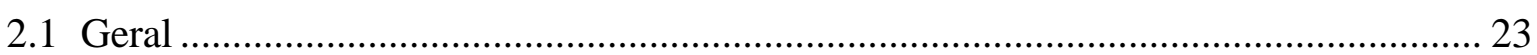

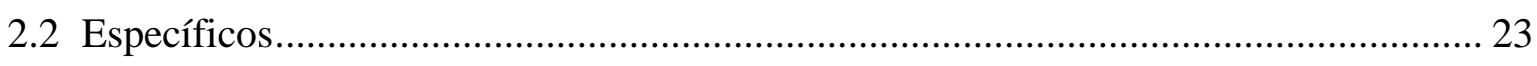

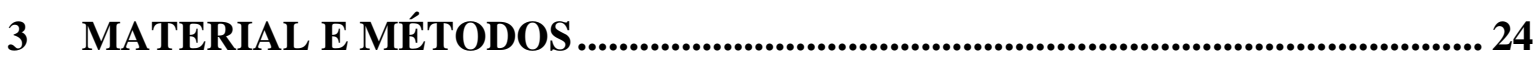

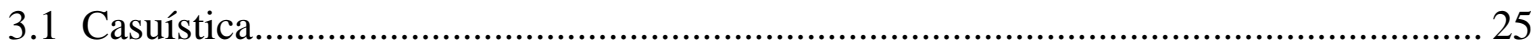

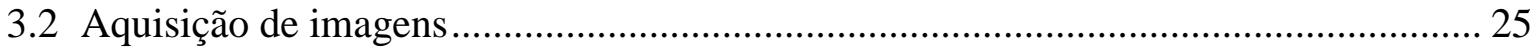

3.3 Processamento e análise das imagens ................................................................... 26

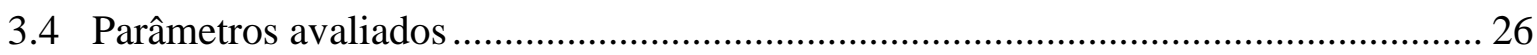

3.5 Metodologia de avaliação................................................................................. 26

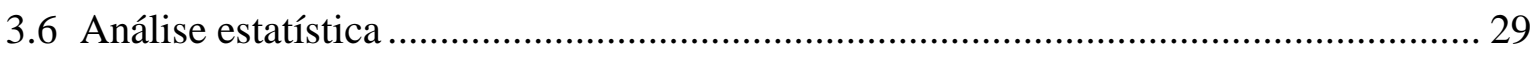

4 RESULTADOS ........................................................................................................... 31

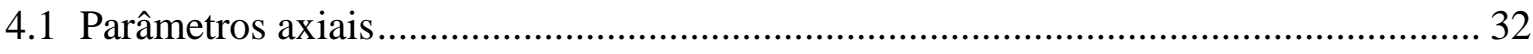

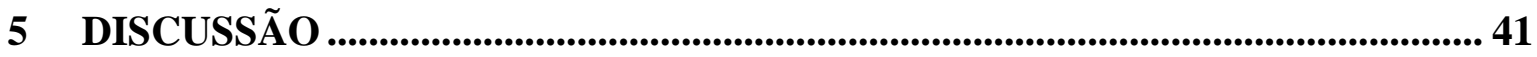

6 CONCLUSÃO ………………………................................................................... 49

REFERÊNCIAS .................................................................................................................... 51

ANEXO A- Parecer Comitê de Ética em Pesquisa ................................................................. 57 
INTRODUÇÃO 


\section{INTRODUÇÃo}

\subsection{Embriologia coluna cervical}

As vertebras cervicais em conjunto com as demais vertebras da coluna vertebral apresentam seu desenvolvimento embrionário a partir de células mesenquimais, que ao final da terceira semana de gestação se diferenciam e iniciam a formação dos somitos. No final da quinta semana de gestação já existem 42 a 44 pares de somitos sendo compostos por 2 regiões, o esclerótomo e o dermomiótomo. As células dos esclerótomos se organizam em 3 grupos principais, sendo o primeiro grupo o que envolve imediatamente a notocorda e é precursor dos corpos vertebrais e da porção do anel fibroso dos discos intervertebrais. $\mathrm{O}$ segundo grupo rodeia o tubo neural e tornar-se o arco posterior da vértebra e por fim, o terceiro grupo que é o formador de células no interior da parede do corpo e está relacionado com o tecido extra-espinal.(figura 1) Após essa etapa, inicia-se a segmentação com a divisão de cada esclerótomo em uma metade cranial e outra caudal. A metade caudal mais densa desenvolve em direção a cranial para formação do centrummesenquimal, que é o primórdio do corpo vertebral. Na 6 semana ao redor do centrum aparece 2 centros de cartilagem (condrificação) que irão se fundir para formar um bloco cartilaginoso na linha média, formando também o aspecto posterior. Em seguida desenvolvem os processos transversos e espinhosos, ficando a notocorda na região central. Na oitava semana os 3 centros cartilaginosos dão origem aos centros de ossificação primários, que aparecerão inicialmente no ponto inferior da coluna torácica seguindo em direção caudal e cranial. (HERKOWITZ et al., 2011). 

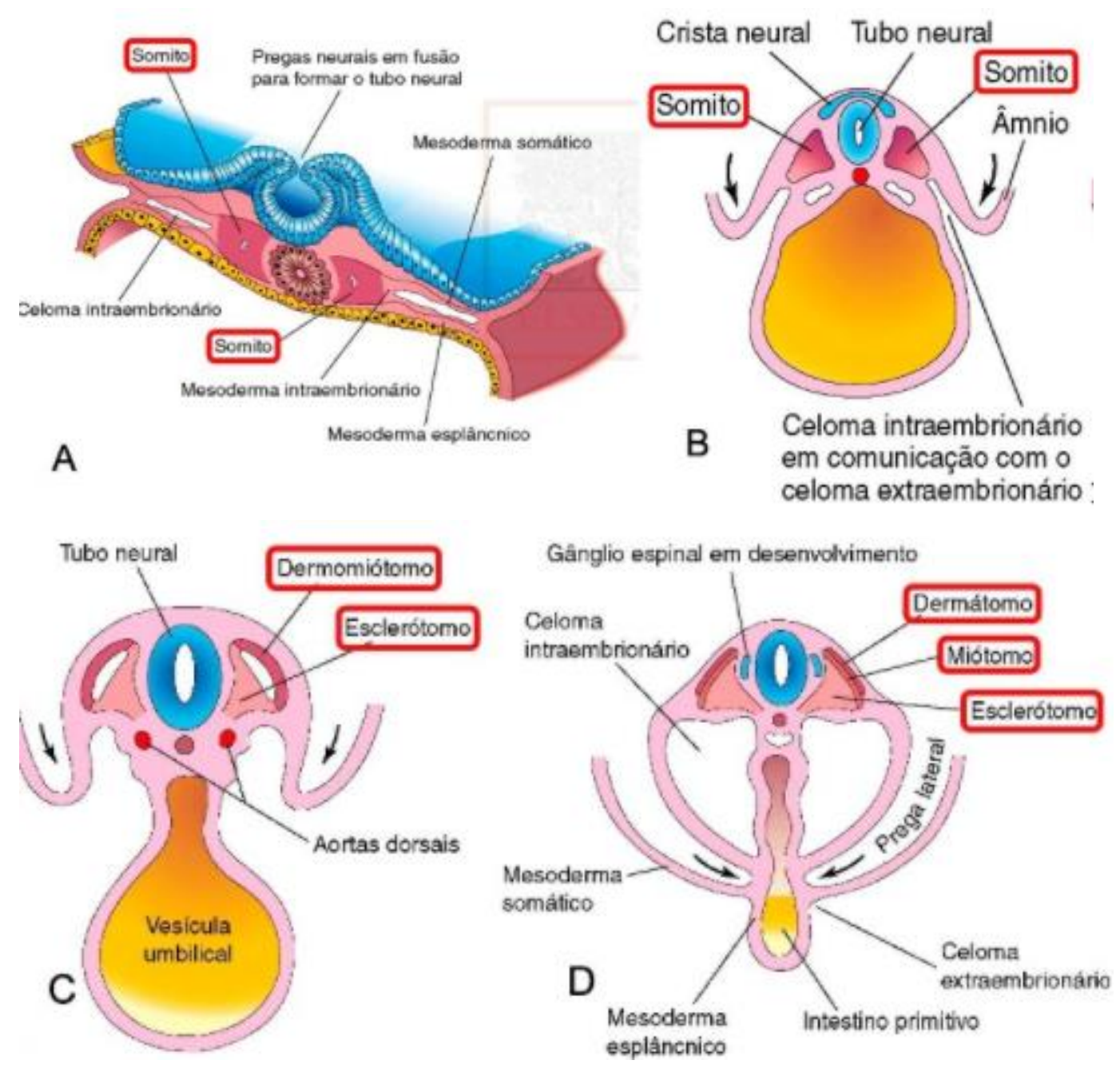

Figura 1 - Cortes transversais do embrião. A e B, note os somitos recém formados. Em C, observe a diferenciação do somito em dermomiótomo e esclerótomo, e em D, a diferenciação do demomiótomo em demátomo e miótomo.

Fonte: (MOORE; PERSAUD; TORCHIA, 2012).

Centros de ossificação secundários desenvolverão após o nascimento. Há cinco centros: uma na ponta da apófise espinhosa, um em cada ponta do processo transverso, e um como anel da epífise nas placas terminais superiores e inferiores dos corpos vertebrais. Este desenvolvimento ocorre até os 15 ou 16 anos de idade, mas, eventualmente, esses centros de ossificação fundirão no meio da terceira década (HERKOWITZ et al., 2011). 


\subsection{Anatomia e fisiologia da coluna cervical}

A coluna cervical é o segmento mais proximal da coluna vertebral, formada por um conjunto de 7 vertebras, com curvatura lordótica no plano sagital, podendo ser divididas em cervical alta, formada pelas 2 primeiras vertebras (atlas e axis) com características anatômicas próprias e que são as responsáveis por 40 a $50 \%$ do movimento de rotação. Já a coluna cervical baixa é formada pelas 5 vertebras cervicais restantes e que apresentam características anatômicas semelhantes entre si, com exceção da última vertebra cervical (C7) que apresenta algumas características de transição com as vertebras torácicas. A cervical baixa é bastante flexível, permitindo movimento em flexão e extensão, inclinação lateral e rotação (WHITE; PANJABI, 1990).

As vertebras cervicais baixas apresentam os seguintes componentes anatômicos ósseos básicos: corpo vertebral, massa lateral, forame transverso, canal medular, lamina posterior, processo espinhoso e pedículo. (figura 2)

\section{Vértebras Cervicais [C4 e C7] Vistas Superiores}

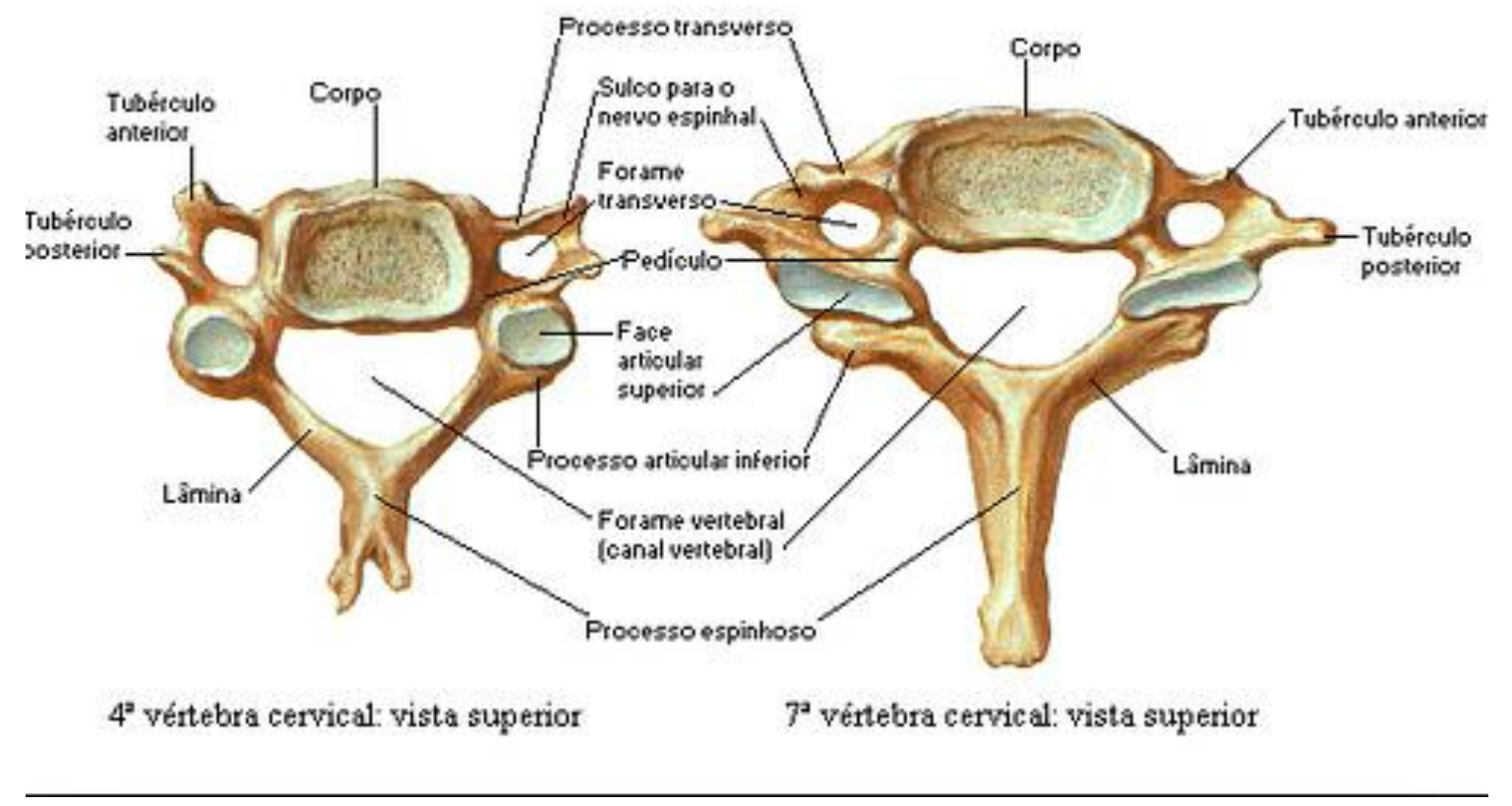

Figura2 - Vértebras Cervicais [C4 e C7].

Fonte: (NETTER, 1999). 
Os corpos vertebrais cervicais, por suportar menos peso, são menores em relação ao tamanho dos arcos vertebrais e forame medular. As bordas laterais da região superior de cada corpo são direcionadas amplamente para cima para formar os processos uncinados que são característicos da região cervical. A característica mais peculiar da vertebra cervical é o forame transverso que perfura os processos transversos e onde está localizada e continda as artérias vertebrais. A artéria vertebral, a veia vertebral e o plexo simpático vertebral passam através do forame transverso (FT) das vértebras cervicais. As artérias vertebrais não passam pelo forame transverso da sétima vértebra cervical (C7). As artérias vertebrais entram pelo FT no nível de C6. (WILLIAMS et al., 1989). Em estudos prévios com cadáveres, o tamanho, o número, e as variações do FT foram medidas e observou-se que o diâmetro do forame diminui de cranial para caudal, sendo o maior diâmetro em $\mathrm{C} 1$ e o menor em $\mathrm{C} 7$. A hipótese é que o diâmetro mais estreito ao nível de C7 nesses estudos deve-se ao fato de que nenhuma artéria vertebral passe dentro do forame neste nível. Alguns estudos demonstraram que os forames transversos da esquerda são discretamente maiores que os forames da direita em todos os níveis, independente do sexo ou raça (TAITZ; NATHAN; ARENSBURG, 1978; ERBIL et al., 2001; CAGNIEet al., 2005; DUAN et al., 2009; EVANGELOPOULOS et al., 2012).

As massas laterais são volumes ósseos localizados posteriormente ao forame vertebral na cervical baixa, que articulam póstero-superiormente com as massas dos níveis adjacentes, formando com tubo de osso. Estudos demonstraram que as massas laterais sofrem uma diminuição de volume de cranial para caudal dos níveis de C5 a C7. Essa região anatômica é muito utilizada como um local comum para a inserção do parafuso durante a fixação interna da coluna cervical (PAIT; McALLISTER; KAUFMAN,1995).

O canal vertebral é formado póstero lateralmente pelas lâminas; antero lateralmente pelos pedículos; e anteriormente pela superfície posterior dos corpos vertebrais.No interior do canal vertebral encontramos a medula espinhal, as raízes dos nervos espinhais, as meninges, o liquor e as estruturas neurovasculares que suprem essas estruturas.O diâmetro sagital médio (anteroposterior) do canal vertebral ósseo varia em função do segmento estudado, sendo de cerca de $21 \mathrm{~mm}$ entre $\mathrm{Cl}$ c C3, 18mm de $\mathrm{C} 4$ a C7. A média das medidas transversas obtidas entre as corticais internas dos pedículos é de aproximadamente $24 \mathrm{~mm}$ na região cervical. Existe grande variação desses valores em função de sexo, raça e constituição dos pacientes, e o diagnóstico de estenose do canal vertebral não deve ser feito isoladamente com base somente nessas medidas (TATAREK, 2005).

As lâminas são estreitas e têm uma borda superior, mais fino. Na sua junção que ocorre na região dorsal, elas formam um processo espinhoso bífida que recebe as inserções 
musculares paravertebrais dorsais. A altura da lâmina de C4 é de aproximadamente 10 a 11 mm, enquanto que a espessura da lâmina em C5 é de cerca de 2 mm(HERKOWITZ et al., 2011).

\section{Vértebras Cervicais [C2-T1], Agrupadas \\ Vista Lateral Direita}

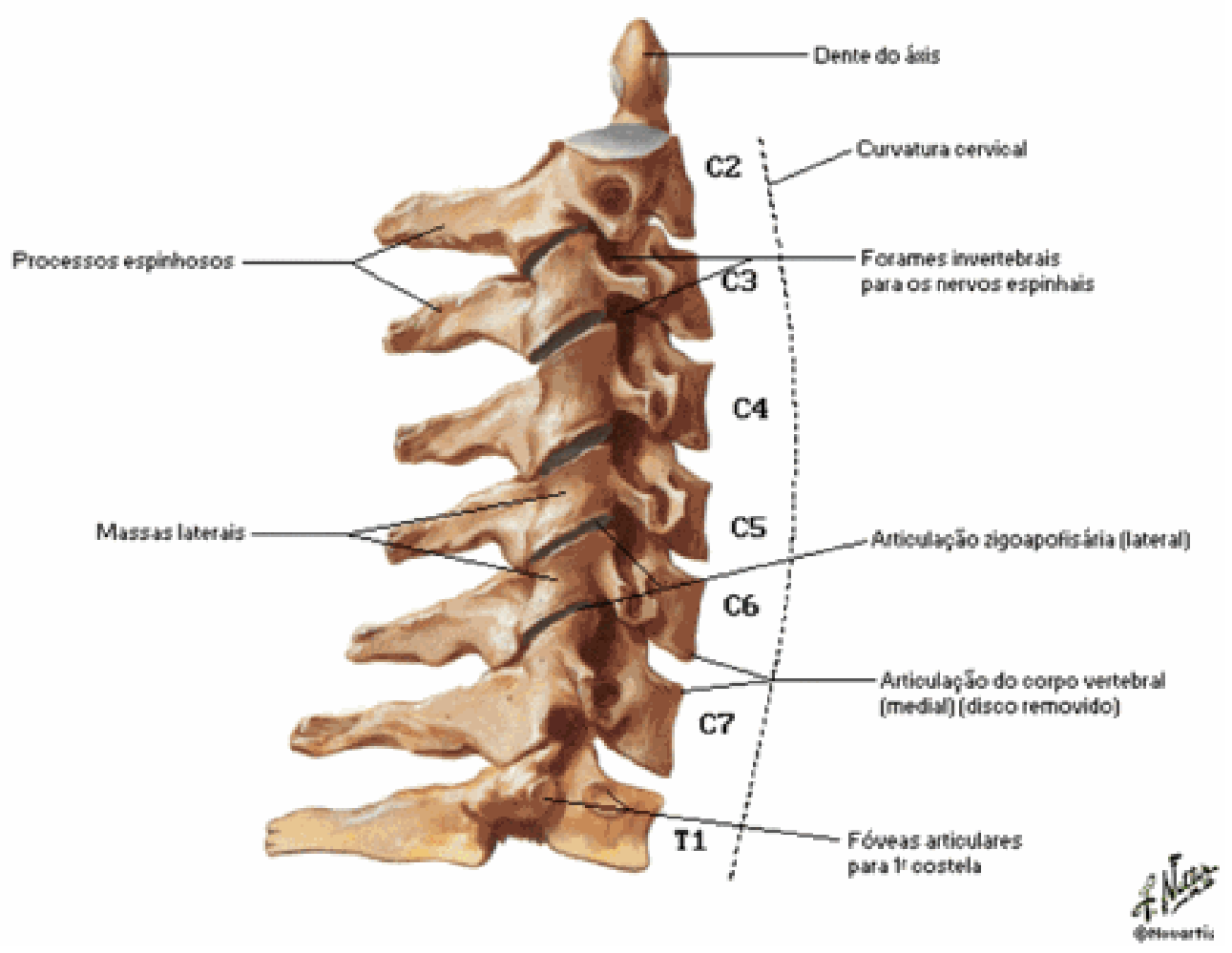

Figura3 - Vértebras Cervicais (C2-T1) agrupadas - vista lateral direita.

Fonte: (NETTER, 1999)

\subsection{Técnicas cirúrgicas coluna cervical}

A estabilização cervical posterior com parafusos de massa lateral limita a artrodese aos segmentos patológicos e permite uma construção posterior rígida que, por sua vez, permite mobilização precoce (WEIDNER, 1994; ROY-CAMILLE et al., 1992). A trajetória é de fundamental importância para os parafusos de massa lateral, pois as raízes nervosas, as artérias vertebrais, as facetas articulares e a medula espinhal estão em risco de sofrerem lesão 
com o posicionamento inadequado. Roy-Camilleet al. (1989), Anderson et al.(1991) e Jeanneret et al.(1991) descreveram técnicas de inserção posterior de parafusos de massa lateral que são comumente utilizadas atualmente. A inserção deve garantir adequadas fixação e segurança, pois estruturas vasculares e neurológicas localizam-se próximas aos pontos de fixação. Heller et al. (1991) compararam as técnicas de inserção de parafusos de Magerl e Roy-Camille e indicaram que a lesão das raízes nervosas e das artérias vertebrais permanece um importante problema durante o procedimento. Eles encontraram que a colocação do parafuso de acordo com Roy-Camille apresentava uma taxa de erro significantemente menor com relação à zona correta de inserção e lesão das raízes nervosas. Os parafusos de Magerl foram melhores com relação à violação das facetas. Nenhuma técnica colocou em risco a medula espinhal ou as artérias vertebrais.

O estudo anatômico de An et al. (1991) observou que o ponto de saída da raiz nervosa encontrava-se na porção anterolateral da faceta superior. Assim, quanto menos lateral ou mais cefálico a trajetória da broca, maior a chance de compressão da raiz nervosa. Anet al. concluíram que o ângulo ideal do orifício é de aproximadamente 15 graus na direção superior e 30 graus na direção lateral para C3-C6, utilizando o ponto de entrada de Anderson (1 mm medial ao centro da massa lateral). Outros estudos mostraram também a importância de uma angulação mais lateral no plano parasagital ou o uso de parafusos mais curtos com menor angulação para evitar as estruturas neurovasculares(XUet al., 1999; XU et al., 1998; EBRAHEIM et al., 1998).

Os dispositivos de placa e parafusos para as massas laterais provaram serem seguros mesmo com a proximidade das estruturas neurovasculares. No entanto, as técnicas de colocação de placa e parafusos posteriores podem estar associadas com problemas potenciais, incluindo a lesão da artéria vertebral, raízes nervosas, facetas articulares e medula espinhal.(XU et al., 1999; XU et al., 1998; EBRAHEIM et al., 1998; GRAHAM et al., 1996; HELLER et al., 1995; JÓNSSON; RAUSCHNING, 1994).

As intervenções cirúrgicas na coluna cervical estão cada vez mais frequentes e associada a modernos métodos de instrumentação. Além do objetivo de corrigir as afecções que geram instabilidade ou compressão cervical, como infecções, tumores, fraturas, deformidades ou doenças degenerativas, busca-se manter e conquistar a maior estabilidade possível desses segmentos da coluna (ONIBOKUN et al., 2009). Das técnicas de instrumentação disponíveis hoje, a fixação transpedicular é umas das mais avançadas e utilizadas para a correção das instabilidades, principalmente da coluna torácica e lombar. $\mathrm{Na}$ fixação do parafuso pedículo cervical, um parafuso é inserido a partir da massa lateral ao 
vertebral corpo, passando todo o interior do pedículo cervical, que atua como uma ponte óssea. Para cada um dos lados, uma haste é colocada para ligar os parafusos e estabilizar o segmento cervical. Alguns estudos biomecânicos demonstram recentemente, que a mesma técnica pode ser utilizada na coluna cervical com pequeno risco, maior estabilidade e maior força de arranchamento comparada com outras técnicas de fixação cervical posterior. (KOTANI et al., 1994; JONES et al., 1997; ABUMI et al., 2000;RHEE et al., 2005; OHNSTON et al., 2006; DUNLAP et al., 2010).No entanto, o método não é usado universalmente devido a seus desafios e riscos. (UĞUR et al., 2000; NEO et al., 2005;) inserção do parafuso pedicular pode ser difícil e arriscado, por causa das dimensões reduzidas do pedículo e pelo potencial de lesões nas estruturas neurovasculares próximas(ALBUMI et al., 1994;UĞUR et al., 2000; NEO et al., 2005). Por isso o sucesso da fixação transpedicular cervical e a diminuição dos riscos de lesão dessas estruturas estão intimamente ligados com o conhecimento e estudo tridimensional por meio de imagens radiológicas da anatomia cervical (XU et al., 1999; UĞUR et al., 2000; REZCALLAH et al., 2001; SAKAMOTO et al., 2004;EVANGELOPOULOS et al., 2012).

\subsection{Método tomográfico de imagem na coluna cervical}

As imagens tomográficas cervicais têm grande importância e utilidade em diversas patologias como na identificação de fraturas e lesões traumáticas, na quantificação de doenças degenerativas, lesões líticas ou blásticas tumorais, discíites e deformidades ou mal formações ósseas. Outra utilidade das imagens tomográficas está na avaliação anatômica óssea dos pacientes para planejamento de possíveis abordagens cirúrgicas.

As principais indicações da tomografia são diagnóstico e estadiamento de fraturas, avaliação de estenose ósseo do canal vertebral, diagnóstico de tumores primários e secundários ósseos, detecção de doenças degenerativas espinhais, diagnóstico de erosões e osteíte na artrite reumatoide e espondiloartropatias, avaliação pós-operatória de pacientes com instrumental metálico, pesquisa de calcificações e quando a Ressonância Magnética é contraindicada ou indisponível.

As desvantagens da tomografia computadorizada incluem a exposição a radiação, os efeitos do cálculo da média do volume parcial, o tempo envolvido na realização de múltiplas delgadas (1,5 a $3 \mathrm{~mm}$ ) secções ao longo de vários corpos vertebrais e intervir discos, 
artefactos raia na coluna cervical causado pelo osso denso do ombro cinto, e as alterações na configuração da coluna vertebral que ocorre entre o movimento sucessivo segmentos(FERNANDE; MACIEL JÚNIOR, 2011).

Alguns estudos utilizando avaliação de imagens tomográficas, conseguiram demonstrar algumas relações e parâmetros do pedículo cervical de C3 a C7 em diversas populações com norte-americanos, chineses, indianos e malaios (ONIBOKUN et al., 2009; YUSOF et al., 2007; RUOFU et al., 2008; GUPTA et al., 2012).

Porém não existe na literatura um estudo da anatomia cervical por meio de imagens tomográficas da população brasileira. 


\section{OBJETIVOS}

\subsection{Geral}

Determinar as características morfométricas e anatômicas dos pedículos das vértebras cervicais de $\mathrm{C} 3$ a $\mathrm{C} 7$, utilizando imagens de tomografia computadorizada.

\subsection{Específicos}

- Comparar nossos resultados com os de estudos previamente reportados;

- Determinar a reprodutibilidade intra e interobservador do método de avaliação empregado;

- Mostrar a importância da tomografia computadorizada como ferramenta na programação pré-operatória de cirurgias da coluna cervical. 


\section{MATERIAIS E MÉTODOS}

\subsection{Casuística}

Trata-se de estudo observacional de um banco de dados prospectivo que foi aprovado pelo Comitê de Ética em Pesquisa do Hospital das Clínicas da Faculdade de Medicina de Ribeirão Preto da Universidade de São Paulo (Oficio n 464/2015; data 05/02/2015; Anexo A). Foi solicitada e concedida a dispensa do termo de consentimento livre e esclarecido da pesquisa por se tratar de um estudo cujo objetivo foi analisar as características anatômicas de estruturas ósseas a partir de imagens de tomografia computadorizada adquiridas na Unidade de Emergência do Hospital das Clínicas da Faculdade de Medicina de Ribeirão Preto. Os exames de imagem foram solicitados para outros fins e a utilização das imagens neste estudo não acarretou prejuízo no tratamento pregresso ou futuro dos pacientes incluídos.

O desenho do estudo baseou-se na avaliação de imagens tomográficas da coluna cervical a partir do nível de C3 até o nível de C7, concentrando as medidas no pedículo cervical. Retrospectivamente, selecionamos imagens tomográficas cervicais de 200 indivíduos (100 do sexo feminino e 100 do sexo masculino) que foram obtidas de pacientes atendidos na Unidade de Emergência do Hospital das Clínicas da Faculdade de Medicina de Ribeirão Preto - USP, no período de janeiro de 2012 a dezembro de 2013. A idade média dos homens foi de $38 \pm 16$ anos (variação 18-83 anos) e a idade média das mulheres foi de $43 \pm 18$ anos (variação 18-85 anos).

Os critérios de exclusão utilizados neste estudo foram idade inferior a 18 anos, cirurgia prévia na coluna vertebral cervical, presença das patologias da coluna vertebral cervical como fraturas, tumores, infecções, espondilite anquilosante e doença hiperostótica familiar (DISH). Já os critérios de inclusão foram a idade superior a 18 anos e a ausência de patologias ou cirurgias prévias na coluna.

\subsection{Aquisição de imagens}


Os exames de imagem foram realizados por meio de um aparelho de tomografia computadorizada (Brilliance CT Big Bore 16-slice, Philips Healthcare, Cleveland, Ohio).

\subsection{Processamento e Análise das imagens}

A reconstrução das imagens do aparelho de tomografia computadorizada foi realizada utilizando-se cortes com uma espessura de $2 \mathrm{~mm}$ para o plano axial e $3 \mathrm{~mm}$ para os planos sagital e coronal. A leitura e reprodução das imagens e bem como suas reconstruções, foram obtidas por meio de programa de imagem OsiriX MD, versão 7.0.2 (Pixmeo SARL, Bernex, Suíça). O programa possui ferramentas de medições lineares e angulares, além de permitir visualizar simultaneamente os 3 planos (sagital, coronal e axial) das imagens. Foram selecionadas as imagens axiais e sagitais dos pedículos cervicais da terceira (C3) a sétima (C7) vértebra cervical. Os parâmetros foram avaliados nos planos sagital e axial.

\subsection{Parâmetros avaliados}

Os parâmetros axiais incluíram a espessura pedicular (EP), o comprimento pedicular axial (CPA), o ângulo pedicular transverso (APT), e a distância do ponto de entrada ao ponto entre a lâmina e o processo espinho (DPE). A intersecção do eixo longitudinal do pedículo e a cortical óssea posterior foi considerado o ponto de entrada do parafuso pedicular. As medidas sagitais envolveram a altura do pedículo (AP) e o ângulo pedicular sagital (APS). Além disso, foram calculadas as porcentagens de pedículos com a espessura inferior a $4 \mathrm{~mm}, 4,5 \mathrm{~mm}$, $5 \mathrm{~mm}, 6 \mathrm{~mm}$ e $7 \mathrm{~mm}$.

\subsection{Metodologia de avaliação}

Dois investigadores independentes realizaram as medidas dos parâmetros relacionados utilizando o programa de imagem OsiriX MD, versão 7.0.2 (Pixmeo SARL, Bernex, Suíça). O primeiro investigador realizou uma avaliação independente adicional com um intervalo de 2 meses entre as medidas, para estimar a confiabilidade intraobservador. 
A lista de terminologia de todos os parâmetros que foram medidos com suas respectivas abreviações e descrições está sumarizada nas Tabelas 1 e 2 . A metodologia de realização das medidas está ilustrada nas Figuras 4 e 5 (figuras obtidas no próprio trabalho). Os parâmetros lineares foram medidos em milímetros $( \pm 1 \mathrm{~mm})$, e os parâmetros angulares foram médios e apresentados em graus. A média e desvio padrão foram calculadas para todas as dimensões pediculares.

O desafio usual de transpor os dados diagnósticos de imagem em informação útil cirurgicamente envolve a dificuldade em delinear estruturas tridimensionais, tal como o pedículo cervical, em duas dimensões. Para obter os dados de maneira mais clinicamente aplicável a partir das imagens de TC, utilizamos a avaliação multiplanar (axial, sagital e coronal) simultânea para a medida de todos os parâmetros.

Tabela1 - Parâmetros medidos nas imagens axiais da TC

\begin{tabular}{|c|c|c|}
\hline Medida & Abreviação & Descrição \\
\hline Espessura pedicular & $\mathrm{EP}$ & Diâmetro mediolateral do pedículo \\
\hline Comprimento pedicular axial & CPA & $\begin{array}{l}\text { Distância da cortical posterior da massa lateral } \\
\text { à parede anterior do corpo vertebral ao longo } \\
\text { do eixo pedicular }\end{array}$ \\
\hline Ângulo pedicular transverso & APT & $\begin{array}{l}\text { Ângulo entre o eixo pedicular transverso e a } \\
\text { linha média do corpo vertebral }\end{array}$ \\
\hline $\begin{array}{l}\text { Distância do ponto de entrada } \\
\text { ao ângulo entre a lâmina e o } \\
\text { processo espinhoso }\end{array}$ & DPE & $\begin{array}{l}\text { Distância entre a projeção do ponto de entrada } \\
\text { do parafuso pedicular na imagem axial e } \\
\text { ângulo formado pela lâmina e o processo } \\
\text { espinhoso }\end{array}$ \\
\hline
\end{tabular}

Tabela 2 - Parâmetros medidos nas imagens sagitais da TC

\begin{tabular}{lll}
\hline Medida & Abreviação & Descrição \\
\hline Altura do pedículo & AP & Diâmetro rostrocaudal do pedículo \\
Ângulo pedicular sagital & APS & Ângulo entre o eixo pedicular sagital e o plano \\
\hline
\end{tabular}


horizontal

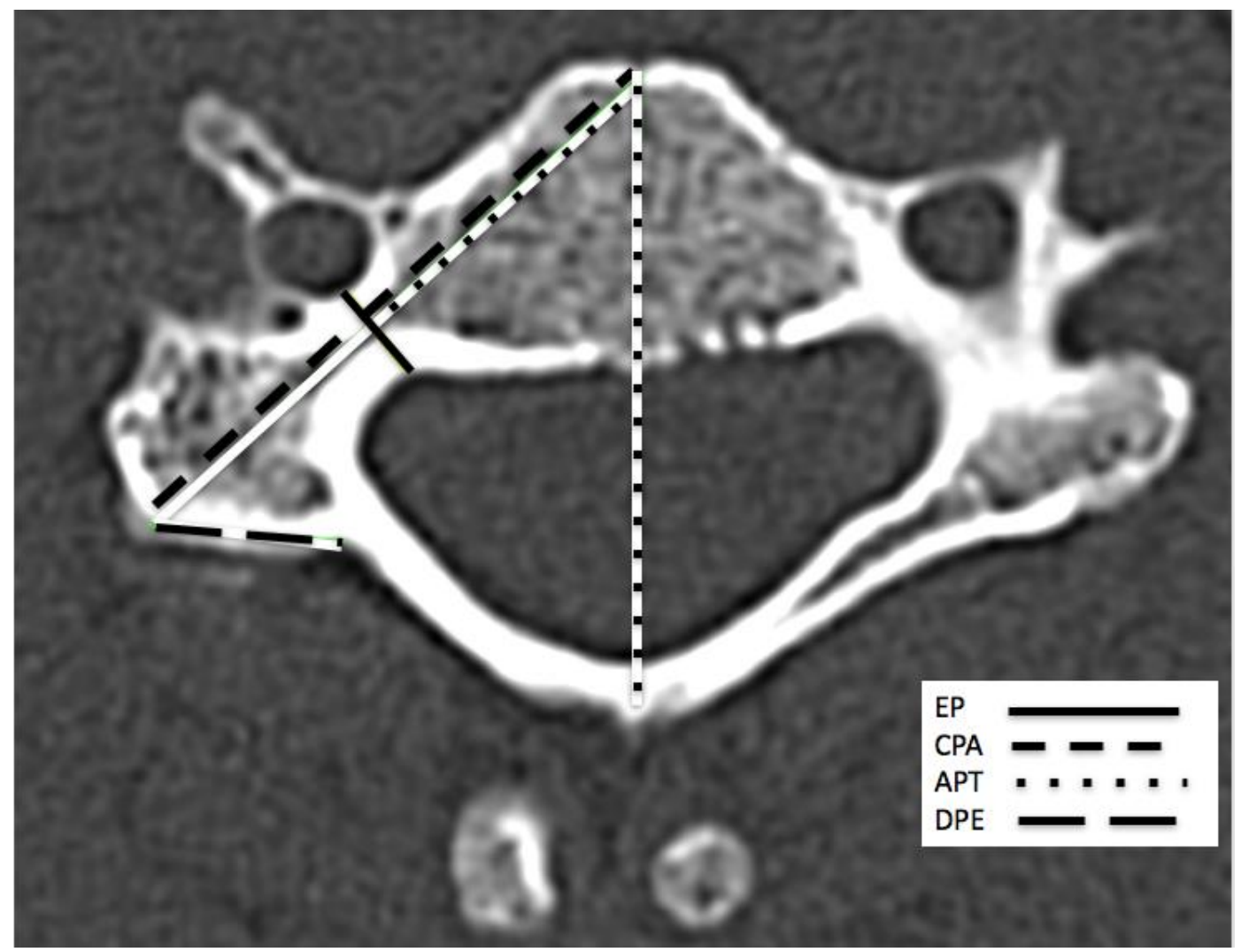

Figura 4 - Imagem da reconstrução axial de tomografia computadorizada ilustrando as medidas realizadas neste plano. 


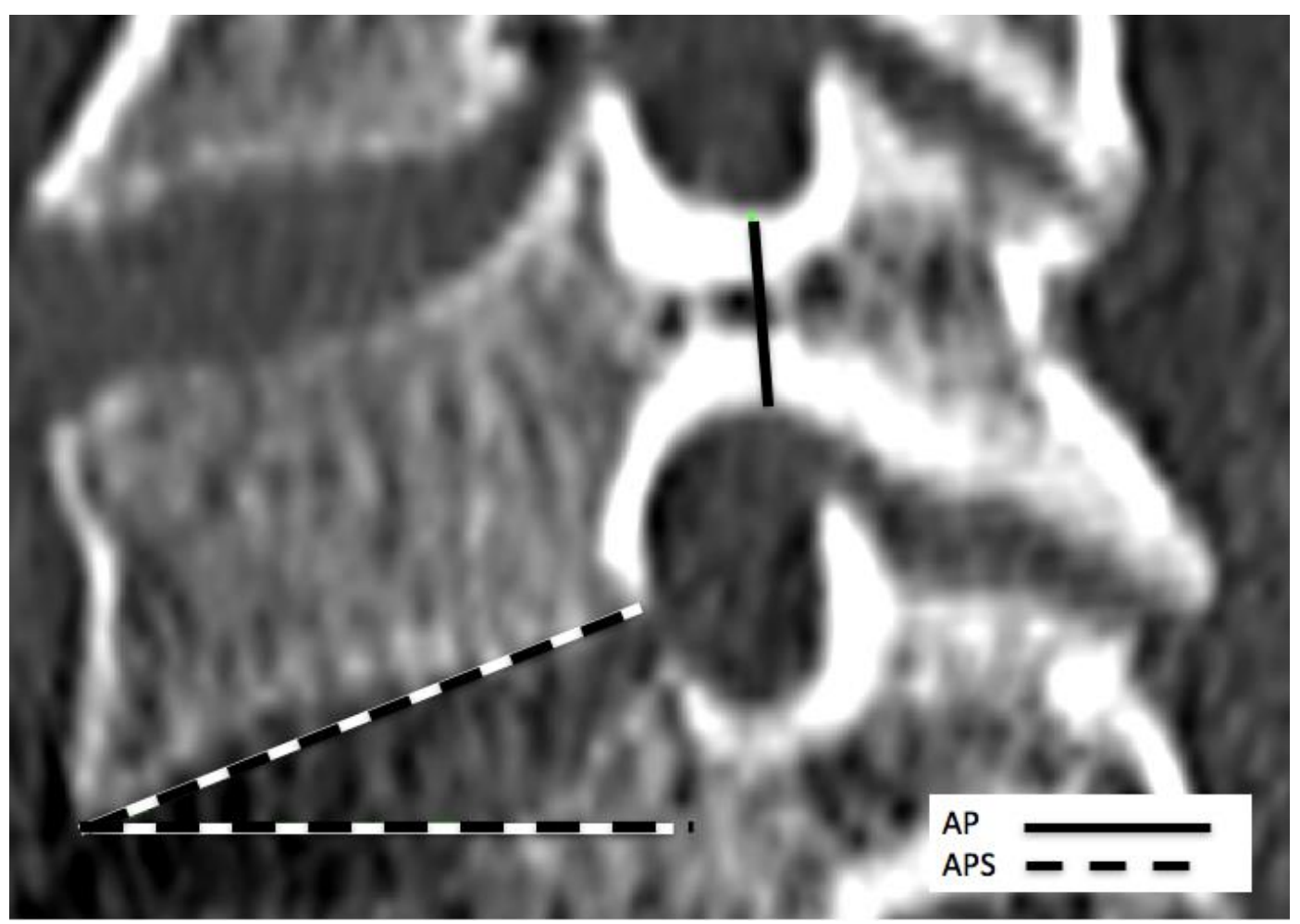

Figura5- Imagem da reconstrução sagital de tomografia computadorizada ilustrando as medidas realizadas neste plano.

\subsection{Análise estatística}

As medidas de TC foram calculadas como médias e desvios padrões. Um total de 2000 pedículos incluindo as vértebras de C3 à $\mathrm{C} 7$ foram avaliados. As médias e desvios padrões dos parâmetros lineares e angulares foram calculados em cada nível para os pacientes do sexo masculino e feminino separadamente (Tabela 3). Dezoito mil medidas foram realizadas e empregadas para determinar a diferença de todos os parâmetros dimensionais e angulares entre os sexos no mesmo nível vertebral. As médias e desvios padrões foram calculadas para as dimensões pediculares, e os valores das medidas foram comparadas utilizando o teste $\mathrm{t}$ de Student.

A confiabilidade intra e interobservador foi estimada utilizando-se o coeficiente de correlação intraclasse (CCI) para as medidas tomográficas. Foi aplicado um modelo de efeito misto de duas vias com consistência concordância. A confiabilidade foi considerada muito fraca para valores entre 0 e 0,2, fraca para valores entre 0,21 e 0,4 , moderada para valores entre 0,41 e 0,6 , substancial ou boa para valores entre 0,61 e 0,8 e quase perfeita ou muito boa para valores entre 0,81 e 1,0 (FLEISS et al., 1973). Nós utilizamos um nível de significância de $5 \%$. Os parâmetros foram armazenados em uma planilha e estudados por meio do 
programa STATA13® (StataCorp. 2013. StataStatistical Software: Release 13. CollegeStation, TX: StataCorp LP).

Tabela 3 - Resumo dos parâmetros lineares e angulares dos pedículos cervicais

\begin{tabular}{cllllll}
\hline & $\mathrm{EP}(\mathrm{mm})$ & $\mathrm{AP}(\mathrm{mm})$ & $\mathrm{CPA}(\mathrm{mm})$ & $\mathrm{APT}\left(^{\circ}\right)$ & APS $\left(^{\circ}\right)$ & DPE $(\mathrm{mm})$ \\
\hline C3 & $4,7 \pm 0,81$ & $6,6 \pm 0,87$ & $32,4 \pm 2,26$ & $45,6 \pm 3,67$ & $16,3 \pm 6,41$ & $8,8 \pm 1,20$ \\
Masc & $5,1 \pm 0,78$ & $6,8 \pm 0,86$ & $33,4 \pm 2,25$ & $45,6 \pm 3,79$ & $15,2 \pm 6,57$ & $8,9 \pm 1,25$ \\
Fem & $4,3 \pm 0,65$ & $6,3 \pm 0,81$ & $31,4 \pm 1,76$ & $45,1 \pm 3,54$ & $17,4 \pm 6,05$ & $8,5 \pm 1,11$ \\
C4 & $4,8 \pm 0,78$ & $6,6 \pm 0,87$ & $31,3 \pm 2,35$ & $46 \pm 3,65$ & $19,9 \pm 5,87$ & $8,4 \pm 1,23$ \\
Masc & $5,2 \pm 0,8$ & $6,9 \pm 0,85$ & $32,3 \pm 2,28$ & $46,3 \pm 3,97$ & $18,8 \pm 5,79$ & $8,6 \pm 1,33$ \\
Fem & $4,5 \pm 0,59$ & $6,3 \pm 0,77$ & $30,4 \pm 2,01$ & $45,7 \pm 3,31$ & $20,8 \pm 5,79$ & $8,3 \pm 1,10$ \\
C5 & $5,2 \pm 0,82$ & $6,5 \pm 1,53$ & $30,9 \pm 2,69$ & $46,4 \pm 4,18$ & $20,3 \pm 5,21$ & $8,4 \pm 1,27$ \\
Masc & $5,6 \pm 0,82$ & $6,8 \pm 1,97$ & $32 \pm 2,92$ & $46,4 \pm 4,57$ & $20,2 \pm 4,57$ & $8,7 \pm 1,33$ \\
Fem & $4,9 \pm 0,66$ & $6,1 \pm 0,76$ & $29,9 \pm 1,97$ & $46,0 \pm 3,75$ & $20,7 \pm 5,78$ & $8,3 \pm 1,18$ \\
C6 & $5,4 \pm 0,86$ & $6,5 \pm 0,86$ & $30,9 \pm 2,55$ & $44,7 \pm 4,73$ & $20,9 \pm 5,30$ & $8,4 \pm 1,16$ \\
Masc & $5,9 \pm 0,84$ & $6,8 \pm 0,84$ & $32,1 \pm 2,35$ & $45,3 \pm 5,46$ & $21,4 \pm 5,83$ & $8,7 \pm 1,14$ \\
Fem & $5,1 \pm 0,71$ & $6,1 \pm 0,79$ & $29,8 \pm 2,19$ & $44,4 \pm 3,86$ & $20,7 \pm 4,69$ & $8,1 \pm 1,09$ \\
C7 & $6,4 \pm 1.00$ & $7.1 \pm 0.90$ & $30.3 \pm 2.61$ & $40.9 \pm 6.19$ & $22.6 \pm 5.81$ & $8.1 \pm 1.00$ \\
Masc & $6.8 \pm 0.97$ & $7.3 \pm 0.83$ & $31.0 \pm 2.24$ & $43.8 \pm 7.16$ & $23.7 \pm 5.59$ & $8.3 \pm 1.04$ \\
Fem & $6.0 \pm 0.84$ & $6.6 \pm 0.83$ & $29.4 \pm 2.49$ & $41.0 \pm 4.64$ & $22.2 \pm 5.94$ & $7.8 \pm 0.90$ \\
\hline
\end{tabular}


Legenda: Masc - gênero masculino; Fem - gênero feminino; EP- Espessura pedicular; AP Altura do pedículo ; CPA - Comprimento pedicular axial; APT - Ângulo pedicular transverso; APS - Ângulo pedicular sagital ; DPE - Distância do ponto de entrada ao ângulo entre a lâmina e o processo espinhoso 


\section{RESULTADOS}

Foram selecionadas aleatoriamente imagens tomográficas cervicais de 200 indivíduos (100 do sexo feminino e 100 do sexo masculino), com um total de 2000 pedículos avaliados, incluindo os níveis vertebrais cervicais de C3 a C7. A idade média dos homens foi de $38 \pm 16$ anos (variação 18-83 anos) e a idade média das mulheres foi de $43 \pm 18$ anos (variação 18-85 anos).

\subsection{Parâmetros axiais}

\section{Espessura pedicular}

A espessura pedicular (EP) média variou de 4,3 a 6,8 $\mathrm{mm}$ (Tabela 3). A menor EP média foi encontrada no nível C3 em ambos os sexos. Assim, nos pacientes do sexo feminino o menor valor encontrado foi de $4,3 \mathrm{~mm}$, enquanto no sexo masculino o menor valor encontrado foi de 5,1 $\mathrm{mm}$. Por outro lado, a maior EP média foi evidenciada em C7, também em ambos os sexos. Enquanto para o grupo do sexo feminino o maior valor médio foi de 6,0 $\mathrm{mm}$, no grupo do sexo masculino o maior valor médio encontrado foi de $6,8 \mathrm{~mm}$.

Foi possível perceber uma tendência ao aumento do valor da EP média conforme avançamos de proximal para distal na coluna cervical (GRÁFICO 1). A EP média encontrada foi menor no grupo de pacientes do sexo feminino quando comparada aos valores encontrados no grupo de pacientes do sexo masculino em todos os níveis da coluna cervical estudados, e esta diferença foi muito significativa em todos os níveis $(\mathrm{P}<0,001)$. 


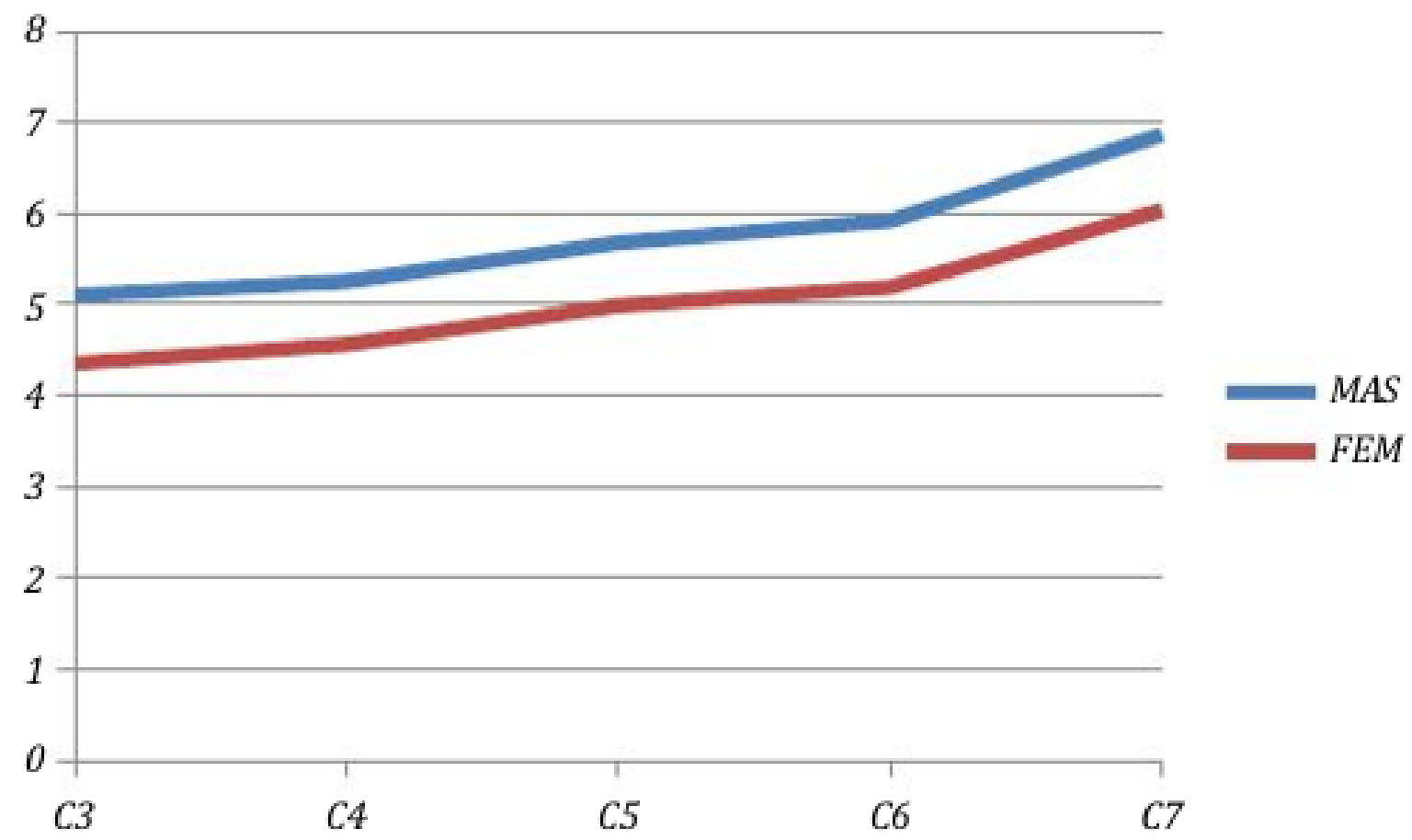

Gráfico1- Ilustrando a variação da espessura pedicular média em milímetros nos níveis vertebrais cervicais estudados em ambos os sexos.

Quando separamos os indivíduos conforme a medida da EP, a porcentagem de indivíduos com EP menor que $4 \mathrm{~mm}$ foi de 7,5\%, no nível C3 do sexo masculino, e $25 \%$ dos indivíduos do sexo feminino no nível C3. Já a porcentagem de indivíduos com EP menor que 4,5 $\mathrm{mm}$ foi de $20 \%$ dos indivíduos do sexo masculino no nível C3, e de $66 \%$ dos indivíduos do sexo feminino no nível C3 (Tabela 4 e Tabela 5).

Tabela 4 - Distribuição da largura do pedículo na amostra masculina

\begin{tabular}{l|c|c|c|c|c}
\hline Masculino & $\mathbf{> 4 m m}$ & $\mathbf{> 4 . 5 m m}$ & $\mathbf{> 5 m m}$ & $\mathbf{> 6 m m}$ & $\mathbf{> 7 m m}$ \\
\hline C3 & $92,5 \%$ & $80 \%$ & $54 \%$ & $12 \%$ & $0 \%$ \\
\hline C4 & $97 \%$ & $79 \%$ & $56 \%$ & $18 \%$ & $1,5 \%$ \\
\hline C5 & $99.5 \%$ & $90 \%$ & $77.5 \%$ & $33 \%$ & $6 \%$ \\
\hline C6 & $99.5 \%$ & $94.5 \%$ & $83.5 \%$ & $47.2 \%$ & $8 \%$ \\
\hline C7 & $100 \%$ & $99.5 \%$ & $97.5 \%$ & $83 \%$ & $38.5 \%$ \\
\hline
\end{tabular}


Tabela 5 - Distribuição da largura do pedículo na amostra feminina

\begin{tabular}{l|c|c|c|c|c}
\hline Feminina & $>4 \mathrm{~mm}$ & $>4.5 \mathrm{~mm}$ & $>5 \mathrm{~mm}$ & $>6 \mathrm{~mm}$ & $>7 \mathrm{~mm}$ \\
\hline C3 & $75 \%$ & $33.7 \%$ & $12.2 \%$ & $1 \%$ & $0 \%$ \\
\hline C4 & $88 \%$ & $52 \%$ & $18 \%$ & $1.5 \%$ & $0 \%$ \\
\hline C5 & $92.5 \%$ & $81 \%$ & $47 \%$ & $5.5 \%$ & $0 \%$ \\
\hline C6 & $97.5 \%$ & $82 \%$ & $58 \%$ & $12 \%$ & $0.5 \%$ \\
\hline C7 & $99.5 \%$ & $95 \%$ & $87.5 \%$ & $49 \%$ & $12 \%$ \\
\hline
\end{tabular}

Comprimento pedicular axial

O comprimento pedicular axial (CPA) médio variou de 29,4 a 33,4 mm (Tabela 3). O menor CPA médio encontrado foi no nível vertebral de C7 em ambos os sexos, sendo no sexo feminino de 29,4 mm e no sexo masculino de 31,0 $\mathrm{mm}$. Por outro lado, o maior CPA médio encontrado foi no nível vertebral de C3 em ambos os sexos, sendo de 31,4 mm no grupo de pacientes do sexo feminino e 33,4 mm no grupo de pacientes do sexo masculino.

O CPA médio foi menor no grupo de pacientes do sexo feminino quando comparado ao do grupo de pacientes do sexo masculino em todos os níveis vertebrais cervicais estudados. A diferença encontrada foi muito significativa em todos os níveis estudados $(\mathrm{P}<0,001)$. Foi possível evidenciar uma tendência a diminuição do CPA médio conforme avançamos distalmente na coluna cervical (Gráfico 2). 


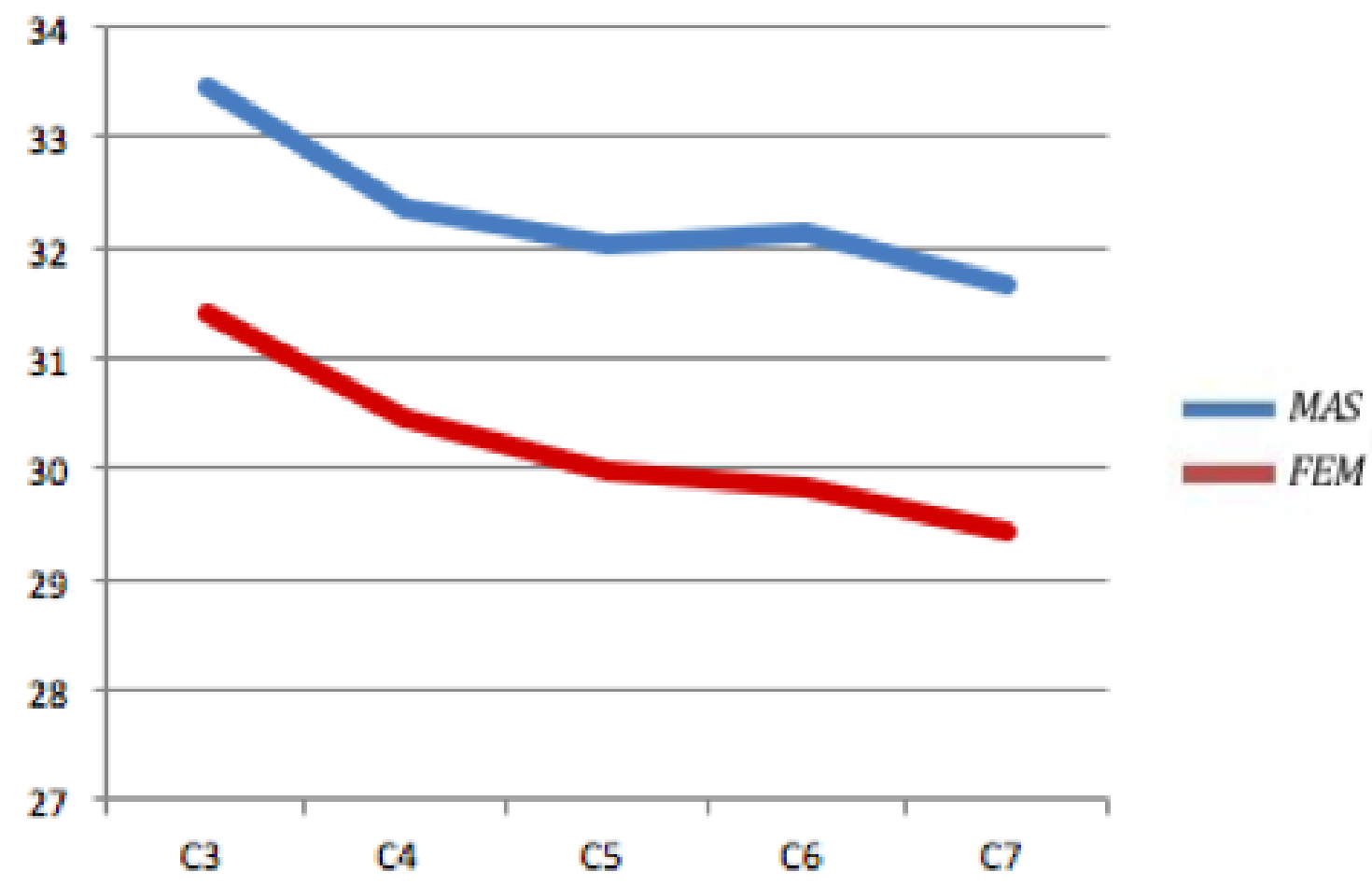

Gráfico2 - Ilustrando a variação do comprimento pedicular axial médio em milímetros nos níveis vertebrais cervicais estudados em ambos os sexos.

Distância do ponto de entrada ao ângulo entre a lâmina e o processo espinhoso

A DPE média variou de 7,8 a 8,9 mm (Tabela 3). A menor DPE média foi encontrada em C7 em ambos os sexos. No grupo do sexo feminino a menor DEP média foi de 7,8 mm, enquanto no grupo do sexo masculino a menor DPE média encontrada foi de $8,3 \mathrm{~mm}$. Os maiores valores médios da DPE foram encontrados no nível vertebral cervical de C3 em ambos os sexos. Enquanto no sexo feminino o maior da DPE média foi de 8,5 mm, no sexo masculino o maior valor foi de $8,9 \mathrm{~mm}$.

Percebemos uma tendência a diminuição dos valores médios da DPE conforme migramos de proximal para caudal na coluna vertebral cervical (Gráfico 3). Não evidenciamos diferença estatística quando comparamos os valores médios de DPE encontrados nos dois grupos. 


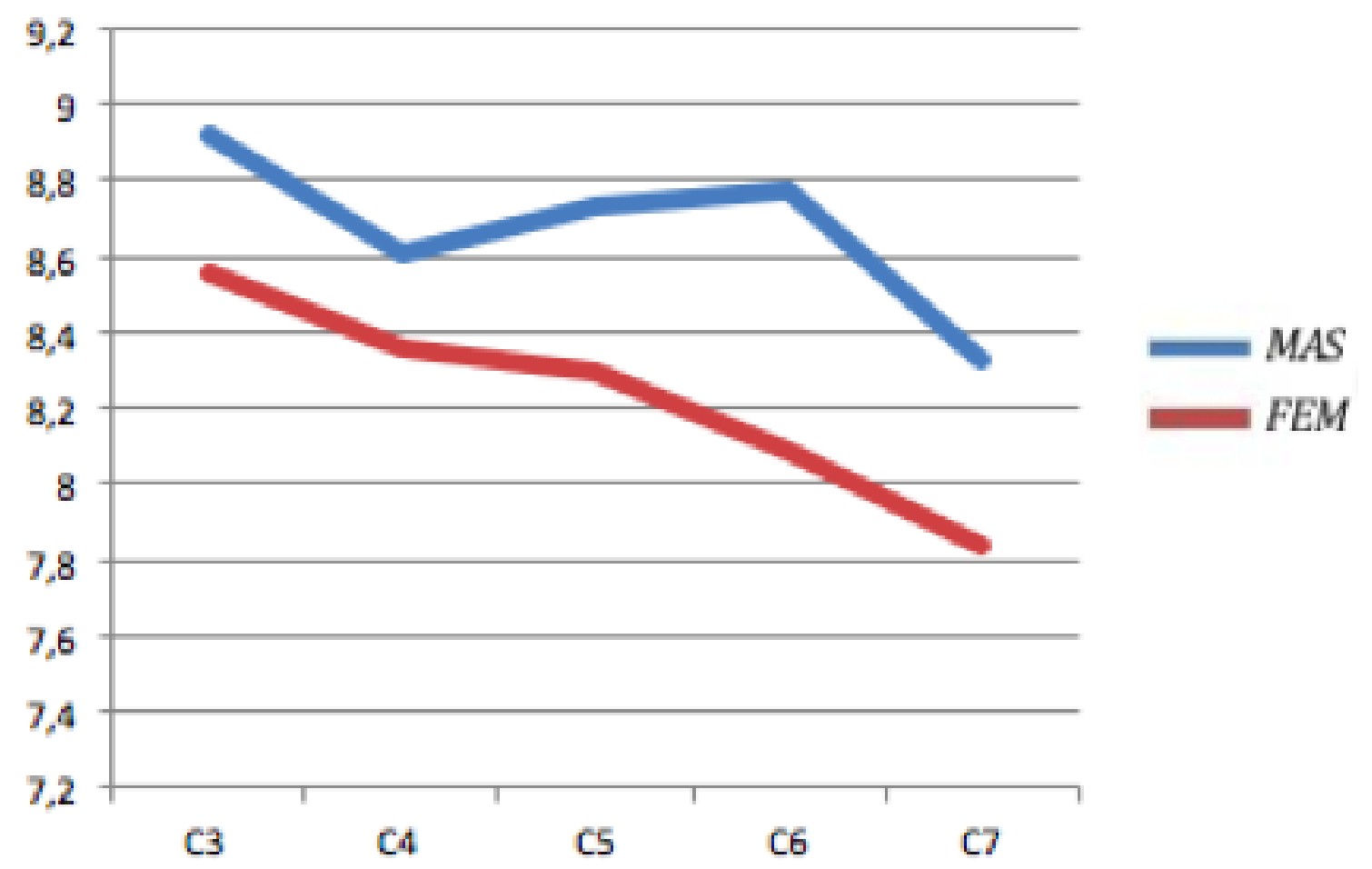

Gráfico3 - Ilustrando a variação da distância em milímetrosdo ponto de entrada ao ângulo entre a lâmina e o processo espinhoso média nos níveis vertebrais cervicais estudados em ambos os sexos.

\section{Ângulo pedicular transverso}

O APT médio variou de $41^{\circ}$ a $46,3^{\circ}$ (Tabela 3). O menor valor do APT médio foi encontrado em C7 em ambos os sexos, sendo de $41^{\circ}$ no sexo feminino e $43,8^{\circ}$ no sexo masculino. Enquanto isso, o maior valor de APT médio encontrado foi de $46^{\circ}$ no sexo feminino e de $46,4^{\circ}$ no sexo masculino, ambos no nível vertebral cervical de C5.

Não conseguimos observar uma tendência de aumento ou diminuição dos valores de APT médio relacionado com a migração para proximal ou distal na coluna vertebral cervical (Gráfico 4). Assim como, também não encontramos diferença estatística significativa em ambos os sexos, em nenhum dos níveis vertebrais estudados. 


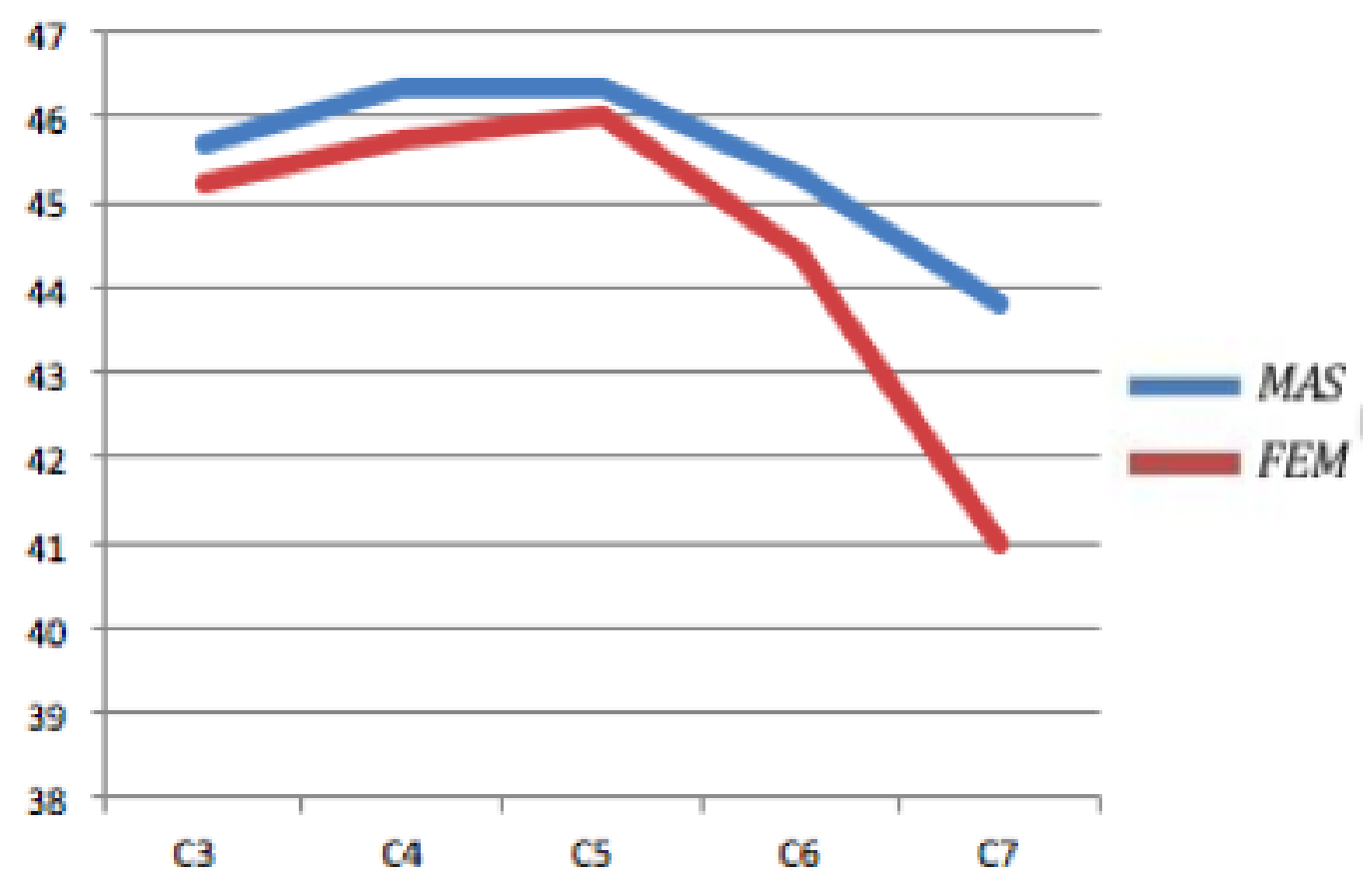

Gráfico4 - Ilustrando a variação angular média em graus do pedículo na posição transversal nos níveis vertebrais cervicais estudados em ambos os sexos.

\subsection{Parâmetros sagitais}

\section{Altura do pedículo}

A AP média variou de 6,1 a 7,3 mm na amostra estudada (Tabela 3). A menor AP média foi encontrada nos níveis C5 e C6 nos pacientes do sexo feminino $(6,1 \mathrm{~mm})$ e nos níveis C3, C5 e C6 nos pacientes do sexo masculino $(6,8 \mathrm{~mm})$. Por ouro lado, a maior AP média foi evidenciada no nível $\mathrm{C} 7$ em ambos os sexos, sendo $6,6 \mathrm{~mm}$ no sexo feminino e 7,3 mm no sexo masculino.

A AP média encontrada foi menor nos grupos do sexo feminino quando comparados aos valores encontrados nos grupos do sexo masculino em todos os níveis, com diferença significativa $(\mathrm{P}<0,001)$. Não observamos aumento gradual da AP média quando avançamos para caudal na coluna vertebral cervical (Gráfico 5). 


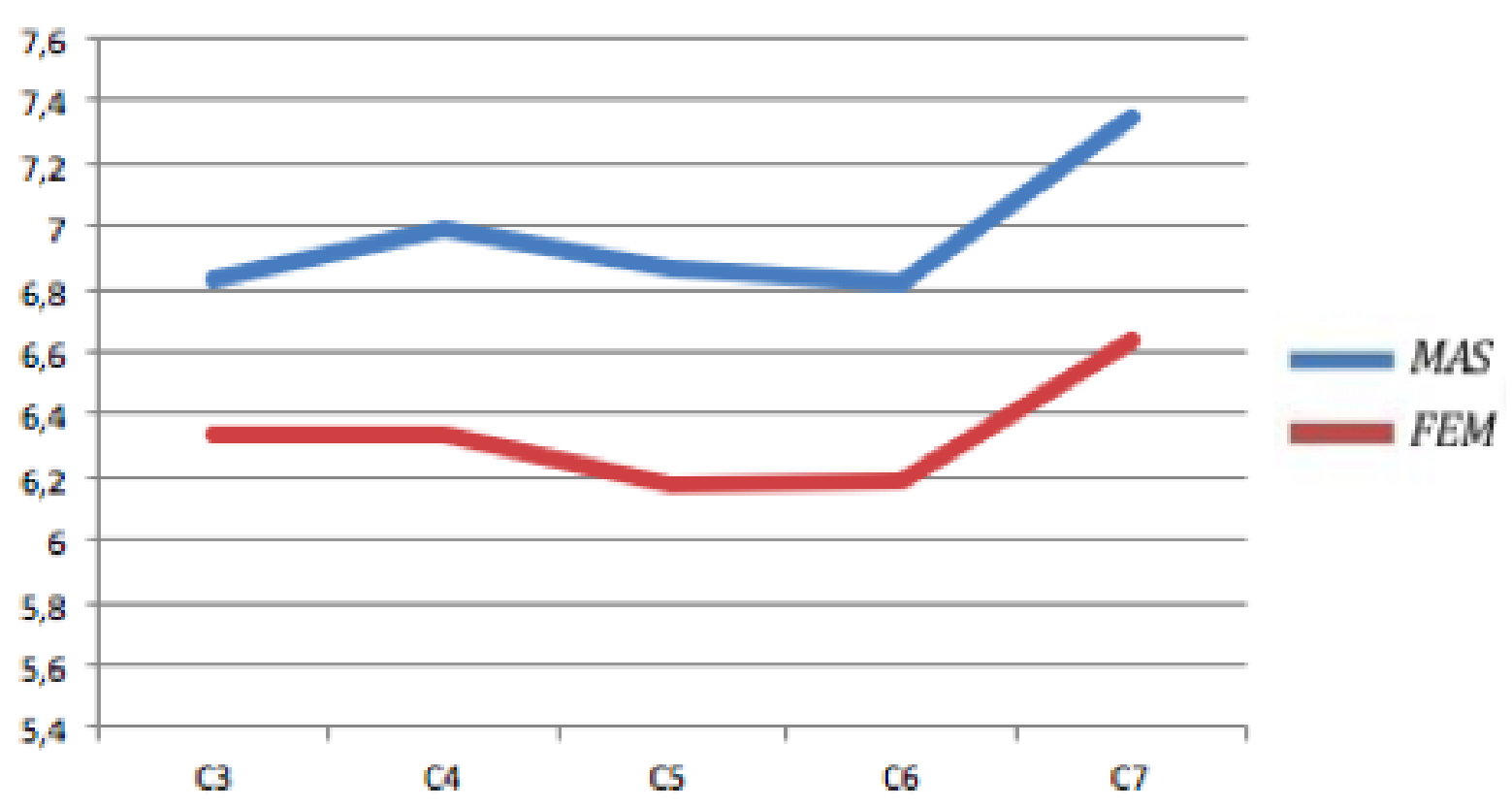

Gráfico 5 - Ilustrando a variação da alturapedicular média em milimitros nos níveis vertebrais cervicais estudados em ambos os sexos.

\section{Ângulo pedicular sagital}

O APS médio variou de $15,2^{\circ}$ a $23,7^{\circ}$ na amostra estudada (Tabela 3 ). O menor APS médio foi encontrado no nível C3 tanto nos pacientes do sexo masculino quanto nos pacientes do sexo feminino. No grupo do sexo feminino o menor APS médio foi $17,4^{\circ}$ e no grupo do sexo masculino o menor APS médio encontrado foi $15,2^{\circ}$. Já o maior APS médio foi evidenciado em C7 em ambos os sexos, sendo o valor no grupo do sexo feminino de $22,2^{\circ}$, e no grupo do sexo masculino de $23,7^{\circ}$ (Gráfico 6). Houve diferença estatística quando comparamos os valores encontrados para os diferentes grupos nos níveis C3 e C4 em ambos os lados, e em C7 no lado direito. 


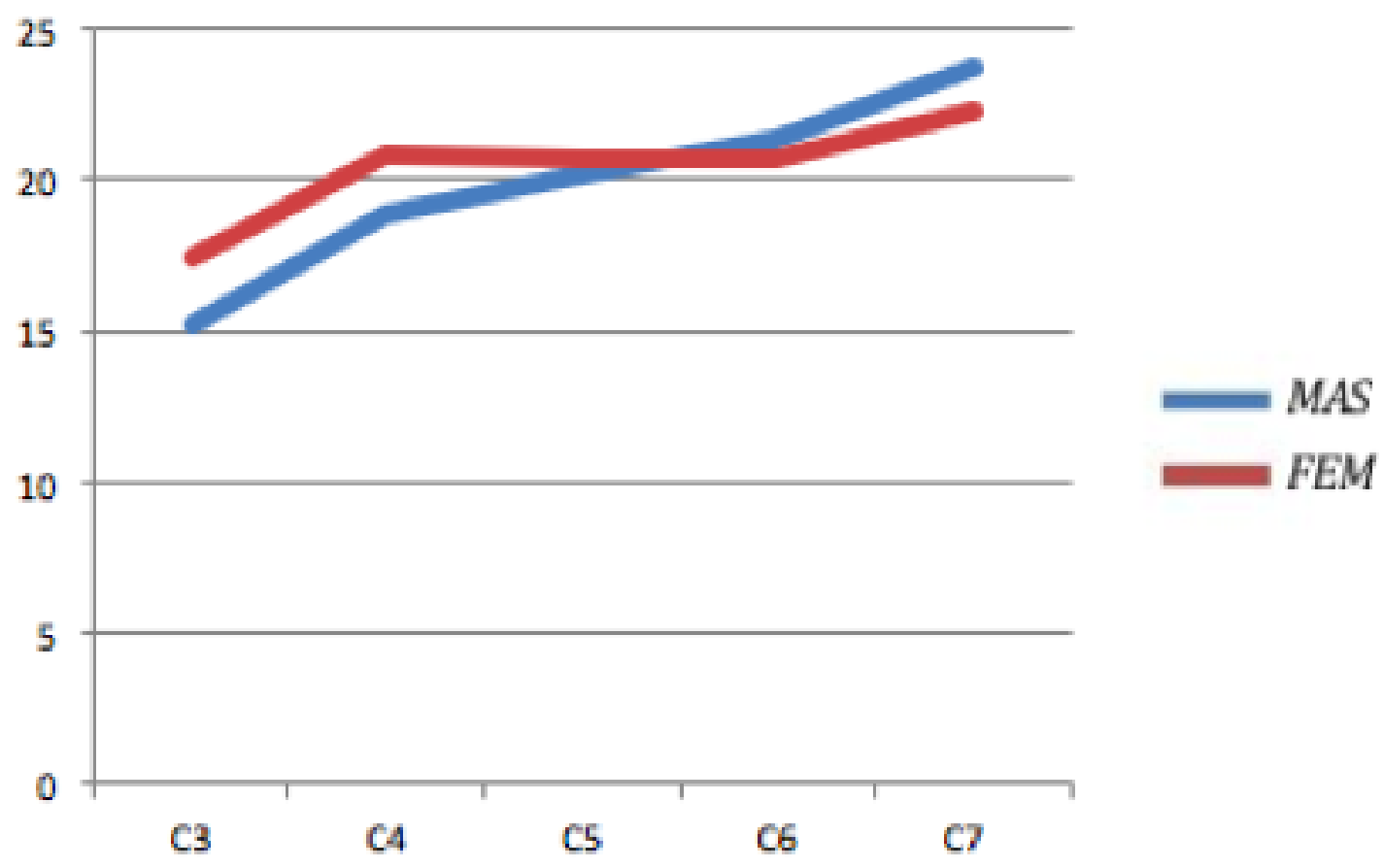

Gráfico 6 - Ilustrando a variação angular média em graus do pedículo na posição sagital nos níveis vertebrais cervicais estudados em ambos os sexos.

\subsection{Confiabilidade intra e interobservador}

A confiabilidade intra e interobservador encontrada foi muito boa para as medidas tomográficas referentes a EP (CCI intraobservador 0,92; interobservador 0,88 e 0,89 ), e boa para as medidas referentes a AP (CCI intraobservador 0,79; ICC interobservador 0,66 e 0,72). Para a variável CPA, a confiabilidade intraobservador encontrada foi boa (ICC 0,76), porém a confiabilidade interobservador variou de moderada a boa (CCI 0,57 e 0,61). Considerando o APT e o APS, a confiabilidade intraobservador observada foi boa (CCI 0,68 e 0,67, respectivamente), no entanto a confiabilidade interobservador foi moderada para o APT (CCI $0,42$ e 0,54$)$ e fraca para o APS (CCI 0,16 e 0,37). As medidas da DPE evidenciaram fraca confiabilidade intraobservador (CCI 0,14), e fraca ou moderada confiabilidade interobservador (ICC 0,20 e 0,49). Os valores de confiabilidade intra e interobservador estão demonstrados na Tabela 6. 
Tabela 6 - Os coeficientes de correlação intraclasse (CCI) com o respectivo intervalo de confiança de 95\% (IC 95\%) para estimativa de confiabilidade intra e interobservador.

\begin{tabular}{llcc}
\hline $\begin{array}{l}\text { Medidas } \\
\text { tomográficas }\end{array}$ & Confiabilidade & CCI & IC 95\% \\
\hline EP & & & $0,90-0,93$ \\
& Intraobservador & 0,92 & $0,59-0,95$ \\
AP & Interobservador & 0,88 & $0,87-0,91$ \\
& & 0,89 & $0,75-0,82$ \\
& Intraobservador & 0,77 & $0,39-0,79$ \\
CPA & Interobservador & 0,66 & $0,67-0,77$ \\
& & 0,73 & $0,71-0,80$ \\
& Intraobservador & 0,76 & $0,53-0,67$ \\
APT & Interobservador & 0,61 & $0,49-0,64$ \\
& & 0,57 & $0,62-0,73$ \\
& Intraobservador & 0,68 & $0,44-0,62$ \\
APS & Interobservador & 0,54 & $0,30-0,51$ \\
& & 0,42 & $0,61-0,97$ \\
DPE & Intraobservador & 0,57 & $-0,12$ até 0,39 \\
& Interobservador & 0,16 & $0,24-0,47$ \\
& & 0,37 & $-0,02$ até 0,28 \\
& Intraobservador & 0,14 & $0,39-0,57$ \\
& Interobservador & 0,49 & $0,04-0,32$ \\
\hline
\end{tabular}

Legenda: EP- Espessura pedicular; AP -Altura do pedículo ; CPA - Comprimento pedicular axial; APT - Ângulo pedicular transverso; APS - Ângulo pedicular sagital ; DPE - Distância do ponto de entrada ao ângulo entre a lâmina e o processo espinhoso 


\section{DISCUSSÃO}

Baseado nos resultados encontrados, esboçamos um perfil anatômico do pedículo cervical na população brasileira, dando ênfase nas diversas medidas e parâmetros que são considerados importantes e até fundamentais para programação e realização de técnicas cirúrgicas. Foi possível também comparar os resultados anatô-micos do pedículo cervical baixo do nosso estudo com estudos populacionais de outros etnias e regiões como estudos realizados nas populações americanas, europeias e asiáticas, sempre com o intuito de viabilizar e programar procedimentos cirúrgicos na prática médica. Segundo Okuyamaet al. a utilização de imagens tomográficas nas medições pediculares em indivíduos vivos oferece informações mais precisas do que estudos com medição manual de espécimes de cadáver. No estudo do grupo de Okuyama, descobriu-se que as medidas dos diâmetros dos pedículos de cadáveres foram significativamente menor do que medições pela tomografia, acreditando-se que ao longo do tempo os cadáveres que são preservados em fluidos de embalsama-mento podem apresentar alterações morfológicas que poderiam afetar as medições (OKUYAMA et al., 1994). Portanto, com os recentes avanços nas modalidades de tomografia computadorizada, as medidas anatômicas do corpo humano tornaram-se mais viáveis e precisas e no nosso estudo buscamos comparar dados dos pedículos de pessoas vivas com outros estudos semelhantes.

Com o objetivo de viabilizar de forma segura e mais ampla a técnica de fixação transpedicular na coluna cervical baixa (C3 a C7), estudos recentes em todo o mundo tem projetado significativo interesse na anatomia tridimensional do pedículo cervical. Essa técnica possibilita fixação das 3 colunas vertebrais (anterior, média e posterior), sendo considerada a mais estável (JONES et al., 1997; KOWALSKI et al., 2000; KOTHE et al., 2004). Kotaniet al.realizou estudo biomecânico comparando a estabilidade de sete técnicas de reconstrução na coluna cervical, e demonstrou que a técnica de parafuso transpedicular confere maior força de arranchamento e maior estabilidade, com a vantagem de aplicar uma abordagem cirúrgica por apenas uma via, a posterior (KOTANIet al., 1994) ou anterior (KOLLER et al., 2008). Porém há limitações a serem consideradas, como a dificuldade de realização da técnica, as diversas estruturas vasculares e neurais que envolvem o pedículo e também o tamanho mínimo necessário do pedículo cervical para a colocação do implante (XU et al., 1999; ABUMI et al., 2000; UĞUR et al., 2000; ONIBOKUN et al., 2009). Yukawaet al.demonstraram em estudo de analise que 620 parafusos pediculares nos níveis de C3 a C6, utilizando flouroscopia intra- 
operatória, que o índice de complicações para esta técnica é baixo, porém é necessário um conhecimentoamplo da anatomia cervical pelos cirurgiões de coluna e devido às possibilidades de riscos graves, este procedimento não deve ser realizado facilmente. Os cirurgiões têm que selecionar melhor o procedimento para cada paciente, como por exemplo evitar esse tipo de procedimento em pacientes com obstrução da artéria vertebral ou com fratura do pedículo, quando história de lesão cervical por trauma. (YUKAWA et al., 2009). Shinet al. ao estudar o pedículo cervical de vertebras de cadáveres humanos em 3 pontos diferentes do trajeto, demonstraram que existe um complexo formato tridimensional com variância linear no plano transversal, com menor espessura cortical na face lateral, o que também aumentaria a periculosidade do procedimento para possíveis lesões da artéria vertebral, enfatizando importância não apenas da espessura do pedículo, mas também para estudo de imagem individualizada do percurso do pedículo. (SHIN et al., 2000).

A largura do pedículo na população brasileira teve como resultado um aumento progressivo das medidas de cranial para caudal em ambos os sexos, as médias variaram de 4,35mm no nível de C3 feminino até 6,83mm no nível de C7 masculino. Em comparação aos sexos, o masculino foi estatisticamente maior que no feminino, para todos os níveis e o lado direito foi discretamente maior que o lado esquerdo, porém sem diferença estatística significativa. Os resultados comparativos com outros estudos, mostrou semelhança dos resultados com os estudos da população europeia e americana. (CHAZONO et al., 2012). Nosso estudo, até o presente momento, foi o que apresentou maior número de pacientes avaliados (200 pacientes), avaliando 1000 vertebras e que comparou com amostras iguais de sexo (100M/ 100F). Onibokunet al. no estudo com 122 (66M/56F) pacientes americanos, encontraram médias de largura do pedículo entre 4,2mm em C3 feminino e 6,9mm em C7 masculino, idênticas ao nosso estudo. (ONIBOKUN et al., 2009). Rao et al. em outro estudo com $98(63 \mathrm{M} / 35 \mathrm{~F})$ pacientes americanos encontrou resultados mais elevados sendo a média variável de 4,8mm em C3 feminino a 7,6mm em C7 masculino. (RAO et al., 2008). Rexcallahet al também estudando $40(28 \mathrm{M} / 12 \mathrm{~F})$ pacientes americanos demonstrou resultados variando de 4,2mm em C3 feminino e 6,4mm em C7 masculino. Todos os estudos com população americana demonstraram as menores larguras em C3 feminino e as maiores em C7 masculino e com exceção do estudo de Rao et al., os resultados foram muito semelhantes (REZCALLAH et al., 2001).

No estudo da população alemã realizado porTomasinoet al. com 127 pacientes $(56 \mathrm{M} / 71 \mathrm{~F})$ apresentou resultado da média variando entre 4,3mm em C3 feminino e 7,2 mm em C7 masculino (TOMASINO et al., 2010). Outro estudo na população alemã realizado por 
Kolleret al. (2008) com 29 pacientes (20M/9F) demonstrou resultados da média variando entre 4,61 mm em C3 feminino a 6,87mm em C7 masculino. Hacker et al. em outro estudo europeu com $54(25 \mathrm{M} / 29 \mathrm{~F})$ pacientes de origem inglesa apresentou como resultado a média de largura do pedículo entre 4,9mm em C3 e 6,6mm em C7,nesse estudo não houve separação de resultados por sexo (HACKER et al., 2008). Reinhold et al. estudando 60 cervicais de cadáveres encontraram como resultados uma média de espessura variando de 5,6mm em C4 e 7,9mm em C7, não houve separação de sexo nesse estudo Quando comparamos com a população europeia, também observamos muita semelhança com nosso estudo e nossos resultados (REINHOLD et al., 2007). E por fim quando avaliamos a população oriental, podemos encontrar resultados diferentes dos apresentados em nosso estudo da população brasileira, com largura do pedículo maior na população japonesa e discretamente maior na população chinesa. Chazonoet al. no estudo com 63 (46M/17F) pacientes japoneses, demonstraram médias de largura do pedículo entre 4,6mm em C3 feminino e 7,0 mm em C7 masculino (CHAZONO et al., 2006). Outro estudo na população japonesa realizado por Sakamoto et al. com 30 pacientes $(18 \mathrm{M} / 12 \mathrm{~F})$ trouxe como resultados, média variando entre 5,5mm em C3 feminino a 7,7mm em C7 masculino. (SAKAMOTOet al., 2004). Ruofuet al. estudando $60(30 \mathrm{M} / 30 \mathrm{~F})$ pacientes chineses registraram resultados variando de 4,4mm em C3 feminino e 6,7mm em C7 masculino (RUOFU et al., 2008). No estudo da população malaia com 40 pacientes $(24 \mathrm{M} / 16 \mathrm{~F})$, Yusofet al. demonstraram resultado da média variando entre 4,6mm em C3 feminino e 6,5 mm em C7 masculino (YUSOF et al., 2007).

Para um parafuso cervical com diâmetro de $3,5 \mathrm{~mm}$, consideraremos a espessura e altura mínimas do pedículo como maior ou igual a 4,5mm, para ser viável o procedimento cirúrgico $(1,11)$. Portanto, assumindo que a largura mínima de 4,5 mm é necessário para aceitar a menor parafuso disponíveis hoje, que é de 3,5 mm, os nossos resultados indicam que a maioria dos pedículos do sexo masculino (80\% em C3, 79\% em C4, 90\% em C5, 94,7\% em C6, e 99,5\% em C7) (Tabela 4) são passíveis de parafuso de fixação. Em comparação com a população masculina, uma percentagem menor de pedículos cervical feminino seria capaz de acomodar um parafuso de 3,5 mm (33,7\% em C3, 52\% em C4, 81\% em C5, 82\% em C6, e 95\% em C7) (Tabela 5). Quando utilizando intervalo de confiança de 95\%, todos os pedículos masculinos apresentaram espessura média acima de $4,5 \mathrm{~mm}$. No entanto, na população feminina os pedículos C3 e C4 do lado esquerdo apresentaram resultado mínimo da média da espessura inferior a 4,5mm, ou seja existe o risco aumentado de parcela dessa população no procedimento de fixação transpedicular, mostrando que estudo tomográfico pré operatório é muito importante nessa população, principalmente quando envolve os níveis de C3 e C4, 
resultado semelhante ao estudo de Onibokunet al.(2009). Gupta et al. encontraram uma média de largura do pedículo na amostra da população indiana variando de 2,9-7,8 mm, sendo que em quase $72 \%$ dos casos, observou-se largura do pedículo maior do que 4,5 mm, dos quais $50 \%$ de todos os casos, era mais do que $5 \mathrm{~mm}$ (GUPTA et al., 2012). Comparando-se com a população asiática, nosso estudo apresentou umaporcentagem menor da população masculina e feminina com pedículos maiores que 4,5mm (resultado masculino variando de $0,5 \%$ em C7 a $20 \%$ em C3 e no feminino de $5 \%$ em C7 a 66,3\% em C3) comparando-se ao estudo de Ruofu et al. (2008) com 60 pacientes na população chinesa (resultado masculino variando de $0 \%$ em C7 a 16,7\% em C4 e no feminino de $0 \%$ a 55\% em C3) e Yusof et al. com 40 pacientes(10) na população malaia (4,2\% em C7 para 16,7\% em C3 nos homens e 7,1\% em C7 para 56,3\% em C3 nas mulheres) foram menores que 4,5 mm.y (YUSOF et al.,2007)

Como a técnica de parafuso transpedicular exige altura e espessura mínimas de segurança para ser realizada, focamos também as medidas na altura do pedículo. $\mathrm{Na}$ população indiana Gupta et al. demonstraram que a altura do pedículo variou de 4.6 a $9.8 \mathrm{~mm}$, onde $88 \%$ dos casos, observou-se ser maior do que $5 \mathrm{~mm}$ (GUPTA et al., 2012). Onibokunet al. obtiveram resultados da altura do pedículo aproximadamente 1 a $2 \mathrm{~mm}$ maior do que a espessura comparando-se em cada nível cervical, indicando que este parâmetro é menos provável de ser um fator limitante para a colocação do parafuso(ONIBOKUN et al., 2009). A altura do pedículo na população chinesa no estudo de Ruofuet al. demonstrou um aumento de cranial para caudal, sendo as menores alturas encontradas em C3 feminino de 5,7mm e em C4 masculino de 6,4mm, e as maiores alturas encontradas em C7 (masculino 7,2mm e feminino 6,9), sendo em média de $0,3 \mathrm{~mm}$ (C7 feminino) até $1,5 \mathrm{~mm}$ (C4 feminino) maiores que a espessura correspondente do mesmo nível(RUOFU et al., 2008). Panjabiet al. também mostraram que a altura pedículo é maior do que a largura do pedículo, evidenciando como a maioria dos estudos que a altura do pedículo tem menor importância que a espessura, quando utilizados para realização de cirúrgica com parafusos transpediculares (PANJABIet al., 1991). Reinhold et al. encontraram como resultados das alturas uma diminuição de cranial para caudal com as médias variando de 7,5mm em C3 a 6,7mm em C6, porém em C7 o pedículonão manteve o mesmo padrãoe apresentou altura de 7,2mm (REINHOLD et al., 2007). Encontramos que estatisticamente as medidas da altura permaneceram semelhantes em todos os níveis, porém no sexo masculino as medidas foram maiores estatisticamente que no sexo feminino. Comparando-se com outros estudos de populações americanas, europeu e americano (CHAZONO et al., 2012), nosso estudo demonstrou grande semelhança com todos. Deduzimos que o parâmetro de altura por apresentar homogeneidade nas medidas e 
valores acima de $5 \mathrm{~mm}$ em todos os níveis e sexo, torna-se um parâmetro seguro e revoga menos preocupações do ponto de vista do planejamento cirúrgico, porém não se pode descartar a avaliação desse parâmetro pelo risco de anormalidades anatômicas possíveis.

O ponto de entrada do parafuso transpedicular calculado em nosso estudo mediu a distância do ponto de transição anatômica da lamina cervical com oprocesso espinhoso até o ponto na massa lateral onde passava a linha de projeção do cálculo do angulo axial, levamos em consideração a ideia de outros estudos que relacionam diretamente o ponto de entrada ou angulo axial do pedículo, como no estudo de Sakamoto et al. que acreditam que a colocação segura do parafuso transpedicular na coluna cervical depende da seleção do ponto de entrada para a inserção do parafuso e na orientação apropriada do parafuso no plano transversal. No estudo de Sakamoto eles concluíram que o ponto de entrada deveria ser mais lateral na massa, para se conseguir um angulo de fixação próximo de 50 graus(SAKAMOTO et al., 2004). Abumiet al. relataram que o ponto de penetração do parafuso transpedicular no córtex posterior era ligeiramente lateral ao centro da massa articular e ligeiramente inferior à margem posterior da superfície articular superior na coluna cervical. Orientando um angulo para a inserção do parafuso de $25^{\circ}$ a $45^{\circ}$ no plano transversal(ABUMI et al., 2000). Ruofuet al. (2008) estudaram o ponto de entrada para inserção do parafuso utilizando $z$ duas distancias e determinaram que o ponto correto de entrada para a inserção do parafuso está localizado em sua grande maioria na área lateral e superior da massa lateral cervical, semelhante ao estudo de Abumiet al. (2000). Jeanneret et al. (1994) recomendam que o ponto de entrada para a inserção do parafuso transpedicular deve estar no meio da massa articular e $3 \mathrm{~mm}$ abaixo da faceta articular superior.

Eles propuseram um ângulo de $45^{\circ}$ medial no plano transversal de C3-C7, também informou que a direção mais comum de violação foi a direção lateral; Por conseguinte, parecia mais seguro para escolher um ângulo de $45^{\circ}$ de inserção (JEANNERET et al., 1994). Nossos resultados demonstraram uma tendência de diminuição da distância do ponto de entrada de cranial para caudal, acompanhando a tendência de diminuição do angulo de projeção axial, demonstrando uma relação entre os dois, ou seja, para conseguir uma angulo maior de projeção do parafuso, deve-se realizar o ponto de entrada mais distante do processo espinhoso, ou em outras palavras, mais lateral na massa lateral. A medida que a distância do processo diminui, de cranial para caudal, o ponto de entrada fica mais central na massa lateral.

As angulações do pedículo no plano axial são importantes parâmetros utilizados na técnica de inserção dos parafusos transpediculares nos dois planos. Abumiet al. sugeriram um angulo de inclinação axial em torno de 25 a 45 graus, no estudos de Jeanneretet al. 
propuseram um ângulo medial de $45^{\circ}$ no plano transversal de $\mathrm{C} 3-\mathrm{C} 7$, uma vez que a violação cortical foi relatada mais na direção lateral(ABUMI et al., 2000; JEANNERET et al., 1994). No artigo de revisão de Chazonoet al. o angulo de inclinação axial obtidos a partir dos dados publicados variou em torno de $45^{\circ}$ a partir de C3 a C6 e diminuiu para cerca de $35^{\circ} \mathrm{em}$ C7(CHAZONO et al., 2012). Reinhold et al. em estudo realizado para avaliar técnicas de parafusos tranpediculares tiveram como resultado do angulo axial variando de $47,6^{\circ}$ em $\mathrm{C} 3$; 50,3 $3^{\circ}$ em C4 ; 49, $3^{\circ}$ em C5; $44^{\circ}$ em C6 e 39, $1^{\circ}$ em C7 (REINHOLD et al., 2007). No estudo dapopulação inglesa de Hacker et al., apresentou como resultado do angulo sagital de 49,7 em C3; 50,3 ${ }^{\circ}$ em $\mathrm{C} 4 ; 50,1^{\circ}$ em $\mathrm{C} 5 ; 45,6^{\circ}$ em C6 e 39,3 em $\mathrm{C} 7$, demonstrando igualdade angular nos níveis de C3 a C5, com diminuição de C6 e o menor angulo em C7 (HACKER et al., 2008). A proporção global de comparação do AIA da população Europeia/Americana em relação a populações asiáticas no estudo de Chanozoet al. foi de 99,6-106,2\%, exceto para o nível C7. Nessa revisão, observou-se ausência de diferença racial substancial do AIA, demonstrando que os dados da população japonesa era consistente com os dados publicados para as populações Europeias / americana, embora a linha de referência do eixo pedicular axial fosse um pouco diferente, dependendo dos dados reportados(CHAZONO et al., 2012). Sakamoto et al. ao estudarem o angulo de inserção do parafuso transpedicular, encontraram resultados mais seguros de $50^{\circ}$ do que a $25^{\circ}$ (SAKAMOTOet al., 2004). No estudo da população chinesa realizado por Ruofu et al. demonstraram que o ângulo transversal do pedículo deve variar de $32^{\circ}$ a $56^{\circ}$ no plano transversal(RUOFU et al., 2008). No estudo da população indiana, Gupta et al. encontraram que média do angulo axial foi de $38,7^{\circ} \pm 2,87^{\circ}$ variando de $33^{\circ}$ a $48^{\circ}$, sendo que $79 \%$ dos casos, esta variou entre $36^{\circ}$ a $44^{\circ}$ (GUPTA et al., 2012). No nosso estudo calculamos que o eixo para mediar o angulo de inclinação axial deveria corresponder ao mesmo eixo do possível parafuso da técnica transpedicular. O resultados demonstraram semelhança na média angular axial nos níveis de C3 a C6, em torno de 43,1 a 46,4 graus, com discreta aumento dos ângulos encontrados de cranial para cauda, com exceção do nível de C7 que apresentou uma pequena diminuição variando de 40,1 a 42,8 graus. Nosso estudo apresentou correspondência com todos os outros estudos onde ocorreu aumento do angulo de cranial para caudal, no qual os ângulos ficaram na faixa de 40 a 50 graus e também apresentou como exceção o angulo de C7, com menor inclinação que os demais.

No estudo de Ruofuet al. no que diz respeito aos ângulos dos pedículos no plano sagital, verificou-se que os pedículos C3 e C4 foram dirigidos superiormente em comparação com a placa terminal vertebral inferior, os pedículos C5 foram paralela a ela, e que pedículos 
C6 e C7 foram inferiormente dirigida, variando de 12,6 em C3 a -5, $6^{\circ}$ em C7 (RUOFU et al., 2008). Reinhold et al. também encontraram com resultados do angulo sagital medidas de 19,4 em C3 a 5,3 em C7 (REINHOLD et al., 2007). Em nosso estudo o ângulos dos pedículos no plano sagital direcionamento dos parafusos sempre foi caudal (inferior) variando de 15,2 em C3 a 23,7 graus em C7. A diferença encontrada entre esses estudos pode estar relacionada mais ao posicionamento do paciente quando realizado a imagem de tomografia, do que uma variação anatômica, uma vez que utilizamos a linha horizontal da imagem para calcular o angulo. A existência de poucos estudos desse parâmetro, impossibilita uma comparação e avaliação mais crítica. Como a técnica de parafuso transpedicular 62 utiliza o imagem intra-operatório, recomendamos utilizar o platô vertebral inferior como parâmetro para o angulo sagital durante o procedimento

Outro parâmetro importante relacionado a técnica cirúrgica do parafuso transpedicular é o comprimento do pedículo, pois se o parafuso for muito longo, pode causar fratura do pedículo (LUDWIG et al., 2000; GUPTA et al., 2012). Gupta et al. encontraram que a média de comprimento do parafusos foi de 32,1 $\pm 1,8 \mathrm{~mm}$ (sexo masculino 32,9 \pm 1,6 $\mathrm{mm}$, sexo feminino de $31,3 \pm 2,0 \mathrm{~mm}$ ), podendo variar de 27,6 a $36,6 \mathrm{~mm}, 84 \%$ dos casos, foi maior do que $30 \mathrm{~mm}$, podendo ser interpretado que em $84 \%$ dos casos, os parafusos com o comprimento até $28 \mathrm{~mm}$ pode ser inserido com segurança na região cervical média e inferior(GUPTA et al., 2012). Abumi et al. encontrou comprimento de parafuso que foi de 20, 22, 24 e $28 \mathrm{~mm}$ (ABUMI et al., 2000). Resultados semelhantes foram observados por Bozbugaet al. que sugeriu comprimento de parafuso variando 22,2-27,7 mm (BOZBUGA et al., 2004). Em outro estudo realizado por Ludwig et al. observou-se valores maiores do comprimento, variando de 35,5 a 37,4mm (LUDWIG et al., 2000). Onibakun et al. encontraram valores globais do comprimento do pedículo variando de 29,9 a 32,9mm, sendo o menor valor de 28,4mm no nível de C3 feminino (ONIBOKUN et al., 2009). O estudo do pedículo cervical na população chinesa realizado por Ruofu et al. encontraram comprimento do pedículo com média variando entre 27,9 e 32,5, sendo o menor comprimento em $\mathrm{C} 4$ feminino e o maior em C7 masculino(RUOFU et al., 2008). Koller et al. demonstraram valores entre 22 e $33 \mathrm{~mm}$ em C3-C7 (KOLLER et al., 2008). Os resultados do nosso estudo demonstraram diferença com os estudos realizados nas outras populações mundiais, sendo o menor valor de 29,0mm em C7 e o maior valor de 33,3mm em C3. A progressão de aumento do comprimento no presente estudo foi de caudal para cranial, ou seja, de C7 para C3, diferente da evolução de comprimento de cranial para caudal dos outros estudos realizados. 
CONCLUSÃO 


\section{CONCLUSÃO}

Nossos resultados foram similares aos resultados previamente relatados na literatura, no entanto a frequência de pacientes com a espessura pedicular inferior a $4,5 \mathrm{~mm}$ na amostra estudada é superior aos valores encontrados em outras populações. Esse achado sugere um aumento no risco durante a tentativa de realizar a técnica de fixação com parafuso transpedicular cervical. Além disso, nossos resultados reforçaram a necessidade de um planejamento pré-operatório adequado. 


\section{REFERÊNCIAS}

ABUMI, K.; SHONO, Y.; ITO, M.; TANEICHI, H.; KOTANI, Y.; KANEDA, K.

Complications of pedicle screw fixation in reconstructive surgery of the cervical spine. Spine (Phila Pa 1976),v.25, n.8, p.962-969, 2000.

ALBUMI, K.; ITOH H.; TANEICHI, H.; KANEDA, K. Transpedicularscrewfixation for traumaticlesionsofthemiddleandlower cervical spine:

descriptionofthetechniquesandpreliminary report. JournalofSpinalDisorders, v.7, n. 1, p.1928, 1994.

AN, H. S.; GORDIN, R.; RENNER, K. Anatomic considerations for plate-screw fixation of the cervical spine. Spine, v.16, n. 10, p.S548-551, 1991.

ANDERSON, P.A.; HENLEY, M.B.; GRADY, M.S.; MONTESANO, P.X.; WINN, H.R. Posterior cervical arthrodesis with AO reconstruction plates and bone graft. Spine (Phila Pa 1976), v.16, (Supl.3): S72-79, 1991.

BOZBUGA, M.; OZTURK, A.; ARI, Z.; SAHINOGLU, K.; BAYRAKTAR, B.; CECEN, A. Morphometric evaluation of subaxial cervical vertebrae for surgical application of transpedicular screw fixation. Spine (Phila Pa 1976), v.29, n.17, p.1876-1880, 2004

CAGNIE, B.; BARBAIX, E.; VINCK, E.; D'HERDE, K.; CAMBIER, D. Extrinsic risk factors for compromised blood flow in vertebral artery: anatomical observations of the transverse foramina from C3 to C7. Surgical and Radiologic Anatomy, 27, n. 4, p. 312-316, 2005 .

CHAZONO, M.; SOSHI, S.; INOUE, T.; KIDA, Y.; USHIKU, C. Anatomical considerations for cervical pedicle screw insertion: the use of multiplanar computerized tomography reconstruction measurements. Journal of Neurosurgery Spine, v. 4, n. 6, p. 472-7, 2006.

CHAZONO, M.; TANAKA, T.; KUMAGAE, Y.; SAI, T.; MARUMO, K. Ethnic differences in pedicle and bony spinal canal dimensions calculated from computed tomography of the cervical spine: a review of the English-language literature. European Spine Journal, v. 21, n. 8, p.1451-1458, 2012.

DUAN, S.; LV, S.; YE, F.; LIN, Q. Imaging anatomy and variation of vertebral artery and bone structure at craniocervical junction.European Spine Journal, v. 18, n. 8, p. 1102-1108, 2009.

DUNLAP, B.J.; KARAIKOVIC, E.E.; PARK, H.-S.; SOKOLOWSKI, M.J.; ZHANG, L.-Q. Load sharing properties of cervical pedicle screw-rod constructs versus lateral mass screw-rod constructs. European Spine Journal, v.19, n.5, p.803-808, 2010.

EBRAHEIM, N. A.; KLAUSNER, T.; XU, R. Safe lateral-mass screw lengths in the RoyCamille and Magerl techniques. An anatomic study. Spine, v.23, n.16, p.1739-1742, 1998.

EBRAHEIM, N. A.; XU, R.; KNIGHT, T.; YEASTING, R.A Morphometric evaluation of lower cervical pedicle and its projection. Spine (Phila Pa 1976), v.22, n. 1, p.1-6, 1997. 
ERBIL, K.M.; SARGON, M.F.; CELIK, H.H.; BASAR, R.; AKSIT, D.A study of variations of TFs of cervical vertebras in human: accessory foramina in shape and number.

Morphologie, v.85, p.23-24, 2001.

EVANGELOPOULOS, D.S.; KONTOVAZENITIS, P.; KOURIS, S.; ZLATIDOU, X.; BENNEKER, L. M.; VLAMIS, J. A.; EFSTATHOPOULOS, N. Computerized tomographic morphometric analysis of the cervical spine. The open orthopaedics journal, v.6, n. 1, p.250-254, 2012.

FERNANDES, J. L.; MACIEL JÚNIOR, F. (eds.). Coluna vertebral. Rio de Janeiro: Elsevier, 2011. Cap. 2. p. 37-40.

FLEISS, J.; COHEN, J. The equivalence of weighted kappa and the intraclass correlation coefficient as measures of reliability. Educational and Measurement Psychological, v. 33, p. 613-619, 1973.

GRAHAM, A.W.; SWANK, M. L.;KINARD, R. E.; LOWERY, G. L.; MOSTRADORES, B.E. Posterior cervical arthrodesis and stabilization with a lateral mass plate. Spine, v.21, n.3, p.323-329, 1996.

GUPTA, R.; KAPOOR, K.; SHARMA, U.M.; KOCHHAR, S.; GARG, R. Morphometry of typical cervical vertebrae on dry bones and CT scan and its implications in transpedicular screw placement surgery. Surgical and Radiologic Anatomy, v.35, n.3, p.181-189, 2012.

HACKER, A. G.; MOLLOY, S.; BERNARD, J. The contralateral lamina: a reliable guide in subaxial, cervical pedicle screw placement. European Spine Journal, v.17, n. 11, p.14571461, 2008.

HELLER, J. G.; SILCOX, D. H.; SUTTERLIN, C. E. Complications of posterior cervical plating. Spine (Phila Pa 1976), v.20, n.22, p.2442-2448, 1995.

HELLER, J.G.; CARLSON, G.D.; ABITBOL, J.J.; GARFIN, S.R. Anatomic comparison of the Roy-Camille and Magerl techniques for screw placement in the lower cervical spine.

Spine (Phila Pa 1976), v.16, (Suppl. 10), p.S552-557, 1991.

HERKOWITZ, H. N.; GARFIN, S. R.; EISMONT, F. J.; BELL, G. R.; BALDERSTON, R. Rothman-Simeone the spine. $6^{\text {th }}$ ed. Philadelphia: Saunders, 2011. v. 1.

JEANNERET, B.; GEBHARD, J.S.; MAGERL, F. Transpedicular screw fixation of articular mass fracture-separation: results of an anatomical study and operative technique. Journal of Spinal Disorders, v.7, n. 3, p. 222-229, 1994.

JEANNERET, B.; MAGERL, F.; WARD, E.H.; WARD, J.C. Posterior stabilization of the cervical spine with hook plates. Spine, v.16, (Suppl.3), p.S56 -63, 1991.

JOHNSTON, T.L.; KARAIKOVIC, E.E.; LAUTENSCHLAGER, E.P.; MARCU, D. Cervical pedicle screws vs. lateral mass screws: uniplanar fatique analysis and residual pullout strengths. The spine journal, v.6, n.6, 667-672, 2006.

JONES, E. L.; HELLER, J. G.; SILCOX, D. H.; HUTTON, W. C. Cervical pedicle screws versus lateral mass screws: anatomic feasibility and biomechanical comparison. Spine, v.22, 
n.9, p.977-982, 1997.

JÓNSSON JR, H.; RAUSCHNING, W. Anatomical and morphometric studies in posterior cervical spinal screw-plate systems. Journal of Spinal Disorders, v.7, n.5, p.429-438, 1994.

KOLLER, H.; HEMPFING, A.; ACOSTA, F.; FOX, M.; SCHEITER, A.; TAUBER, M.; HOLZ, U.; RESCH, H.; HITZL, W. Cervical anterior transpedicular screw fixation. Part I: study on morphological feasibility, indications, and technical prerequisites. European Spine Journal, v.17, n.4, p.523-538, 2008.

KOTANI, Y.; CUNNINGHAM, B.W.; ABUMI, K.; MCAFEE, P.C. Biomechanical analysis of cervical stabilization systems: an assessment of transpedicular screw fixation in the cervical spine. Spine (Phila Pa 1976), v.19, n.22, p.2529-2539, 1994.

KOTHE, R.; RÜTHER, W.; SCHNEIDER, E.; LINKE, B. Biomechanical analysis of transpedicular screw fixation in the subaxial cervical spine. Spine, v.29, n.17, p.1869-1875, 2004.

KOWALSKI, J.M.; LUDWIG, S.C.; HUTTON, W.C.; HELLER, .J.G. Cervical spine pedicle screws: a biomechanical comparison of two insertion techniques. Spine (Phila Pa 1976), v. 25, n. 22, p.2865-2867, 2000.

LUDWIG, S.C.; KRAMER, D.L.; BALDERSTON, R.A.; VACCARO, A.R.; FOLEY, K.F.; ALBERT, T.J. Placement of pedicle screws in the human cadaveric cervical spine: comparative accuracy of the three techniques. Spine (PhilaPa 1976), v.25, n.13, p.1655$1667,2000$.

MOORE, K.L.; PERSAUD, T.V.N.; TORCHIA, M.G. Embriologia clínica 9. ed. Rio de Janeiro: Elsevier; 2012.

NEO, M.; SAKAMOTO, T.; FUJIBAYASHI, S.; NAKAMURA, T. The clinicalriskof vertebral arteryinjuryfrom cervical pediclescrewsinserted in degenerativevertebrae. Spine (PhilaPa 1976), v.30, n. 24, p.2800-2805, 2005.

OAKLEY, P.A.; HARRISON, D.D.; HARRISON, D.E.; HAAS, J.W. Evidence-based protocol for structural rehabilitation of the spine and posture: review of clinical biomechanics of posture (cbp) publications. The Journal of the Canadian Chiropractic Association, v.49, n.4, p.270-296, 2007.

OKUYAMA, K.; SATO, K.; ABE, E.; ONUMA, S.; ISHIKAWA, N. Vertebral pedicle diameter as determined by computed tomography: inaccuracies observed by direct measurement of cadaveric lumbar spine. Skeletal Radiology, v.23, n.7, p.551-553, 1994.

ONIBOKUN, A.; KHOO, L.T.; BISTAZZONI, S.; CHEN, N.F.; SASSI, M. Anatomical considerations for cervical pedicle screw insertion: the use of multiplanar computerized tomography measurements in 122 consecutive clinical cases. The Spine Journal,v.9, n.9, p.729-734, 2009.

PAIT, T. G.; McALLISTER, P. V.; KAUFMAN, H. H. Quadrant anatomy of the articular pillars (lateral cervical mass) of the cervical spine. Journal of Neurosurgery, v.82, n.6, p.1011-1014, 1995. 
PANJABI, M.M.; DURANCEAU, J.; GOEL, V.; OXLAND, T.; TAKATA, K. Cervical human vertebrae: quantitative three-dimensional anatomy of the middle and lower regions. Spine (Phila Pa 1976),v.16, n.8, p.861-869, 1991.

RAO, R.D.; MARAWAR, S.V.; STEMPER, B.D.; YOGANANDAN, N.; SHENDER, B.S. Computerized tomographic morphometric analysis of subaxial cervical spine pedicles in young asymptomatic volunteers. The Journal of Bone and Joint Surgery, v.90, n.9 p.19141921, 2008.

REINHOLD, M.; MAGERL, F.; RIEGER, M.; BLAUTH, M. Cervical pedicle screw placement: feasibility and accuracy of two new insertion techniques based on morphometric data. European Spine Journal, v.16, n.1, p.47-56, 2007.

REZCALLAH, A.T.; XU, R.; EBRAHEIM, N.A.; JACKSON, T. Axial computed tomography of the pedicle in the lower cervical spine. American Journal of Orthopedics (Belle Mead, N.J.), v.30, n.1, p.59-61, 2001.

RHEE, J.M.; KRAIWATTANAPONG, C.; HUTTON, W.C. A comparison of pedicle and lateral mass screw constructs stiffnesses at the cervicothoracic junction: a biomechanical study. Spine (Phila Pa 1976), v.30, n.21, p. E636-640, 2005.

ROY-CAMILLE, R.; SAILLANT, G.; BERTEAUX, D.; SERGE, M.A. Method of posterior screw placement techniques. In: MCKIBBIN, B. (ed.). Early management of spinal injuries: recent advances in orthopaedics. Edinburgh: Churchill-Livingstone, 1989. p.5789.

ROY-CAMILLE, R.; SAILLANT, G.; LAVILLE, C.; BENAZET, J.P. Treatment of lower cervical spinal injuries-C3 to C7. Spine (Phila Pa 1976), v.17, (Suppl 10): p.S442-446, 1992.

RUOFU, Z.; HUILIN, Y.; XIAOYUN, H.; XISHUN, H.; TIANSI, T.; LIANG, C.; XIGONG, L. CT evaluation of cervical pedicle in a Chinese population for surgical application of transpedicular screw placement. Surgical and Radiologic Anatomy: SRA, v.30, n.5, p.389396, 2008.

SAKAMOTO, T.; NEO, M.; NAKAMURA, T. Transpedicular screw placement evaluated by axial computed tomography of the cervical pedicle. Spine (Phila Pa 1976), v.29, n.22, p.2510-2514, 2004.

SHIN, E.K.; PANJABI, M.M.; CHEN, N.C.; WANG, J.L. The anatomic variability of human cervical pedicles: considerations for transpedicular screw fixation in the middle and lower cervical spine. European Spine Journal, v.9, n.1, p.61-66, 2000.

TAITZ, C.; NATHAN, H.; ARENSBURG, B. Anatomical observations of the foramina transversaria. Journal of Neurology, Neurosurgery \& Psychiatry, v.41, n. 2, p.170-176, 1978.

TATAREK, N. E. Variation in the human cervical neural canal. The Spine Journal, v.5, n.6, p.623-631, 2005.

TOMASINO, A.; PARIKH, K.; KOLLER, H.; ZINK, W.; TSIOURIS, A.J.; STEINBERGER, J.; HÄRTL, R. The vertebral artery and the cervical pedicle: morphometric analysis of a critical neighborhood. Journal of Neurosurgery Spine, v.13, n.1, p.52-60, 2010. 
UĞUR, H.C.; ATTAR, A.; UZ, A.; TEKDEMIR, I.; EGEMEN, N.; CAĞLAR, S.; GENÇ, Y.Surgicalanatomicevaluationofthe cervical pedicleandadjacent neural structures.

Neurosurgery, v.47, n. 5, p. 1162-8, 2000. UĞUR

WEIDNER, A. Lateral mass screw placement techniques. In: SHERK, H. H. (ed.). The cervical spine: an atlas of surgical procedures. Philadelphia: JB Lippincott Company, 1994. p. $163-75$.

WEINSTEIN, S.L. The pediatric spine: principles and practice. New York: LWW, 1993. p.3-66.

WHITE, A. A.; PANJABI, M.M. (eds.). Clinical biomechanics of the spine. 2.ed. Michigan: Lippincott, 1990.

WILLIAMS, P. L.; GRAY, H. Gray's anatomy. 37.ed. New York: Churchill Livingstone, 1989.

XU, R.; EBRAHEIM, N.A.; KLAUSNER, T.; YEASTING, R.A. Modified Magerl technique of lateral mass screw placement in the lower cervical spine: an anatomic study. Journal of Spinal Disorders,v.11, n.3, p.237-240, 1998.

XU, R.; HAMAN, S.P.; EBRAHEIM, N.A.; YEASTING, R.A. The anatomic relation of lateral mass screws to the spinal nerves: a comparison of the magerl, anderson, and an techniques. Spine (Phila Pa 1976), v.24, n.19, p.2057-2061, 1999.

XU, R.; KANG, A.; EBRAHEIM, N.A.; YEASTING, R.A. Anatomic relation between the cervical pedicle and the adjacent neural structures. Spine (Phila Pa 1976), v.24, n.5, p.451454, 1999.

YUKAWA, Y.; KATO, F.; ITO, K.; HORIE, Y.; HIDA, T.; NAKASHIMA, H.; MACHINO, M. Placement and complications of cervical pedicle screws in 144 cervical trauma patients using pedicle axis view techniques by fluoroscope. European Spine Journal, v.8, n.9, p.1293-1299, 2009.

YUSOF, M.I.; MING, L.K.; ABDULLAH, M.S. Computed tomographic measurement of cervical pedicles for transpedicular fixation in a Malay population.

Journaloforthopaedicsurgery (Hong Kong), v.15, n.2, p.187-190, 2007. 


\section{ANEXO A}

Ribeirâo Preto, 05 de fevereiro de 2015

Oficio $n^{\circ} 464 / 2015$

$\mathrm{CEP} / \mathrm{MGV}$

Prezado Senhor,

O trabalho intitulado "ESTUDO MORFOMETRICO DA COLUNA VERTEBRAL CERVICAL" - Versäo 2, de 10/11/2014, foi analisado "AD REFERENDUM" pelo Comitē de Etica $\mathrm{cm}$ Pesquisa, c enquadrado na categoria: APROVADO, bem como o Termo de Consentimento Livre e Esclarecido Maiores de 18 anos - Versāo 2, de 10/11/2014, de acordo com o Processo HCRP $n^{\circ} 14224 / 2014$.

De acordo com Carta Circular $n^{\prime \prime}$ 003/2011/CONEP/CNS, datada de 21/03/2011, o sujeito de pesquisa ou ser representante, quarido for o caso, deverá rubricar todas as folhas do Termo de Consentimento Livre e Esclarecido - TCLE - apondo sua assinatura na ultima do referido Termo; o pesquisador responsável deverá da mesma forma, rubricar todas as folhas do Termo de Consentimento Livre e Esclarecido - TCLE - apondo sua assinatura na última página do referido Termo.

Este Comite segue integralmente a Conferência Internacional de Harmonizaçao de Boas Práticas Clinicas (IGH-GCP), bem como a Resoluçáo $n^{\circ}$ 196/96 CNS/MS,

Lembramos que devem ser apresentados a este CEP, o Relatório Parcial e o Relatónio Final da pesquisa.

$$
\begin{gathered}
\text { Atenciosamente. } \\
\text { DR. MARCIA GUMARAES VILLANOVA } \\
\text { Coordenadora do Comite de Etica em } \\
\text { Pesquisa do HCRP e da FMRP-USP }
\end{gathered}
$$

Ilustrissimo Senhor

\section{ANDERSON LUIS DO NASCIMENTO}

Depto. de Biomecânica, Medicina e Reabilitaçāo do Aparelho Locomotor

HOSPITAL DAS CLINICAS DA FACULDADE DE MEDICINA DE RIBEIRÃO PRETO DA UNIVERSIDADE DE SÃO PAULO Campus Universitário - Nonte Alegre Comite de Ética em Pesquisa do HCRP O FMRP-USP

FWA-00002733; RE-00002186 e Registro PEJCONEP $\pi^{\circ} 5440$

(16) $3602-2228$ 